TRANSACTIONS OF THE

AMERICAN MATHEMATICAL SOCIETY

Volume 355, Number 12, Pages 4699-4721

S 0002-9947(03)03010-1

Article electronically published on July 28, 2003

\title{
CAUSAL COMPACTIFICATION OF COMPACTLY CAUSAL SPACES
}

\author{
FRANK BETTEN
}

\begin{abstract}
We give a classification of causal compactifications of compactly causal spaces. Introduced by Ólafsson and Ørsted, for a compactly causal space $G / H$, these compactifications are given by $G$-orbits in the BergmanŠilov boundary of $G_{1} / K_{1}$, with $G \subset G_{1}$ and $\left(G_{1}, K_{1}, \theta\right)$ a Hermitian symmetric space of tube type. For the classical spaces an explicit construction is presented.
\end{abstract}

\section{INTRODUCTION}

A causal symmetric space is a symmetric space $(G, H, \tau)$ consisting of a (connected) Lie group $G$, an involutive automorphism $\tau$ of $G$, and a closed subgroup $H$ with $G_{0}^{\tau} \subset H \subset G^{\tau}$ (here $G_{0}^{\tau}$ is the identity component of $G^{\tau}:=\{g \in G \mid \tau(g)=g\}$ ) carrying a $G$-invariant causal structure. In general, a causal structure on a manifold $M$ is the attachment of a cone $C_{x} \subset T_{x} M$ to every point $x \in M$. We always assume our cones to be closed, convex and regular, i.e. proper $\left(C_{x} \cap-C_{x}=\{0\}\right)$ and generating $\left(C_{x}-C_{x}=T_{x} M\right)$. With $\mathfrak{g}$ the Lie algebra of $G$, and the decomposition $\mathfrak{g}=\mathfrak{h}+\mathfrak{q}$ corresponding to $\tau$, we can identify the tangent space at the identity coset $T_{e H} G / H$ of the homogenous space $G / H$ with $\mathfrak{q}$. The $G$-invariant causal structures are now in one-to-one correspondence with $H$-invariant cones $C$ in $\mathfrak{q}$. Restricting our attention to semisimple Lie algebras $\mathfrak{g}$ or to groups $G$ and irreducible symmetric spaces, the irreducible symmetric Lie algebras $(\mathfrak{g}, \mathfrak{h}, \tau)$ corresponding to a causal symmetric space are classified (Ól 90, HÓ 96, p. 89]) and can be divided into three classes. For that, let $\theta$ be a Cartan involution commuting with $\tau$, and $\mathfrak{g}=\mathfrak{k}+\mathfrak{p}$ the corresponding Cartan decomposition; then the subclass of compactly causal spaces is characterized by the existence of a cone with $C \cap \mathfrak{k} \neq \emptyset$ and $C \cap \mathfrak{p}=\{0\}$. $c$-duality, $\mathfrak{g}^{c}:=\mathfrak{h}+i \mathfrak{q}$, defines a correspondence between compactly causal Lie algebras $(\mathfrak{g}, \mathfrak{h}, \tau)$ and noncompactly causal Lie algebras $\left(\mathfrak{g}^{c}, \mathfrak{h}, \tau\right)$. As a third subclass we can single out the intersection of these two classes, the spaces with a self-dual algebra, called causal symmetric spaces of Cayley type.

Definition 1.1 (ÓØ 97]). Let $M$ be a causal $G$-space. A causal compactification of $M$ is a pair $(N, \Phi)$ that satisfies the following conditions:

(1) $N$ is a compact causal $G$-space.

(2) The map $\Phi: M \rightarrow N$ is causal, i.e. if $m \mapsto C_{m}$ is the causal structure on $M$ and $n \mapsto D_{n}$ that on $N$, then $(d \Phi)_{m}\left(C_{m}\right) \subset D_{\Phi(m)}$ for every $m \in M$.

Received by the editors October 7, 1996

2000 Mathematics Subject Classification. Primary 53C35; Secondary 32M15. 
(3) $\Phi$ is injective and $G$-equivariant, i.e. $\Phi(g \cdot m)=g \cdot \Phi(m)$, for every $g \in G$ and every $m \in M$.

(4) $\Phi(M)$ is open and dense in $N$.

Our aim in this article is to construct causal compactifications for compactly causal spaces. The compact space will always be the Bergman-Šilov boundary $\check{S}_{1}$ of a bounded symmetric domain of tube type. In fact, this boundary is a homogeneous space with an invariant causal structure ( $\mathrm{Ka} 89$ ). More precisely, if we choose $\left(G_{1}, H_{1}, \eta\right)$ to be of Cayley type - this implies that $G_{1} / K_{1}$ is Hermitian symmetric of tube type (HÓ 96, Remark 2.6.9]) — there is a parabolic subgroup $P^{\prime}$, with $H_{1} \subset P^{\prime}$, such that $\check{S}_{1} \simeq G_{1} / P^{\prime}$ and the canonical projection $\Phi: G_{1} / H_{1} \rightarrow G_{1} / P^{\prime}$ is causal (and of course $G_{1}$-equivariant). The basic strategy is now to find an involution $\sigma$ of $G_{1}$, commuting with $\theta$ and $\eta$, such that $(G, H, \tau):=$ $\left(\left(G_{1}^{\sigma}\right)_{0}, H_{1} \cap\left(G_{1}^{\sigma}\right)_{0}, \eta \mid\left(G_{1}^{\sigma}\right)_{0}\right)$ is a compactly causal subspace of $\left(G_{1}, H_{1}, \eta\right)$, i.e. its causal structure is defined by restricting the compactly causal structure of $G_{1} / H_{1}$. If $G \cap P^{\prime}=H$, we get by use of $\Phi$ an embedding $G / H \hookrightarrow G_{1} / P^{\prime}$, and it remains to show that $\Phi(G / H)$ is (open) dense. This can be done using Matsuki's classification of $\left(G_{1}^{\sigma}, P_{\min }\right)$-double cosets, $P_{\min }$ a minimal parabolic subgroup of $G_{1}$. Indeed, there are finitely many open double cosets $G w_{i} P_{\min }$, the $w_{i}$ being elements of an appropriate Weyl group, and it is possible to prove that $G P^{\prime}$ contains all of them, for $P_{\min } \subset P^{\prime}$ suitably chosen.

Of course, in general the fixed point group $G_{1}^{\sigma}$ is only reductive, and we therefore define

Definition 1.2. Let $(G, H, \tau)$ be a causal symmetric space with $G$ reductive. Then $(G, H, \tau)$ is weak compactly causal if there exists an $H$-invariant, closed, convex and regular cone $C \subset \mathfrak{q}$ such that $C \cap \mathfrak{k} \neq \emptyset$ and $C \cap \mathfrak{p}=\{0\}$.

For semisimple $G$ this implies the space is compactly causal by definition (HÓ 96 , p. 76]).

In Theorem 5.1 and Theorem [5.9] we give conditions which ensure that the sketched strategy works. Using these theorems, it is possible to construct causal compactifications for most of the compactly causal spaces - eventually with an additional central extension as explained above — listed in Ól 90, HÓ 96, p. 89]. We summarize our results on causal compactifications of weak compactly causal spaces in the following three tables.

TABLE I. Theorem 5.1 construction

$(\mathfrak{g}, \mathfrak{h})$

$(\mathfrak{s}(\mathfrak{u}(p, q) \oplus \mathfrak{u}(p, q)), \mathfrak{s u}(p, q))$

$(\mathfrak{u}(2 p, 2 q), \mathfrak{s p}(p, q))$

$(\mathfrak{u}(p, q), \mathfrak{s o}(p, q))$

$(\mathfrak{s o}(2, n-1) \oplus \mathfrak{s o}(2), \mathfrak{s o}(1, n-1))$

$\left(\mathfrak{e}_{6(-14)} \oplus \mathfrak{s o}(2), \mathfrak{f}_{4(-20)}\right)$

$\left(\mathfrak{s o}^{*}(2 n) \oplus \mathfrak{s o}^{*}(2 n), \mathfrak{s o}^{*}(2 n)\right)$

$(\mathfrak{s p}(n, \mathbb{R}) \oplus \mathfrak{s p}(n, \mathbb{R}), \mathfrak{s p}(n, \mathbb{R}))$

$(\mathfrak{s o}(2, n), \mathfrak{s o}(1, n))$

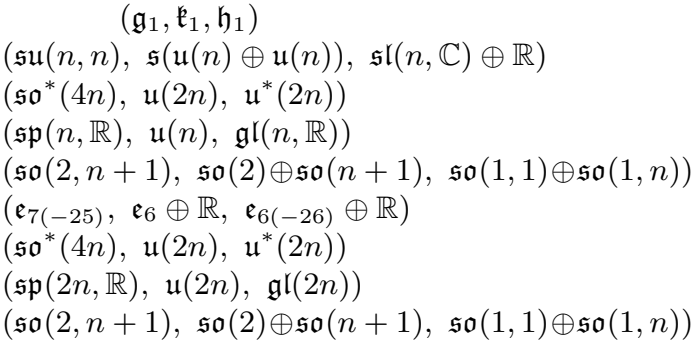


TABLE II. Theorem [5.9] construction

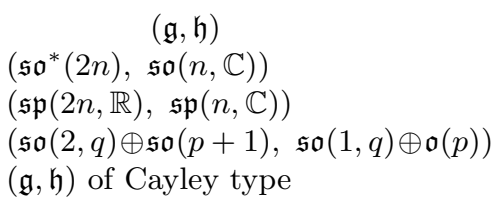

Results by Makarevič ( Ma 73]) together with some simple dimension estimates show that the remaining compactly causal spaces have no causal compactification as symmetric orbits by imbedding them in a Bergman-Šilov boundary, cf. Proposition 6.1

TABle III. No compactification by Proposition 6.1

$$
\begin{array}{lc}
\quad(\mathfrak{g}, \mathfrak{h}) & \multicolumn{1}{c}{\text { parameters }} \\
(\mathfrak{s o}(2, n), \mathfrak{s o}(p, 1) \oplus \mathfrak{s o}(1, q)) & \min (p, q) \geq 2,(p, q) \neq(2,2),(3,3) \\
\left(\mathfrak{e}_{6(-14)}, \mathfrak{s p}(2,2)\right) & \\
\left(\mathfrak{e}_{7(-25)}, \mathfrak{s u}^{*}(8)\right) & \\
(\mathfrak{s o}(2, n) \oplus \mathfrak{s o}(2, n), \mathfrak{s o}(2, n)) & n=5 \text { or } n \geq 7 \\
\left(\mathfrak{e}_{6(-14)} \oplus \mathfrak{e}_{6(-14)}, \mathfrak{e}_{6(-14)}\right) & \\
\left(\mathfrak{e}_{7(-25)} \oplus \mathfrak{e}_{7(-25)}, \mathfrak{e}_{7(-25)}\right) &
\end{array}
$$

We remark that in the same manner as we use Matsuki's double coset decomposition to prove $\Phi(G / H)$ is dense, density of the image and the decomposition into orbits for other open symmetric orbits in $R$-spaces $G / H \rightarrow G_{1} / P^{\prime}$, cf. Ma 73 for a list, can be investigated.

This article is the first step towards a detailed study of the Hardy spaces on compactly causal spaces as introduced by Hilgert, Ólafsson and Ørsted ([HÓØ]). Roughly speaking, these are made up of holomorphic functions on certain open domains in $G_{\mathbb{C}} / H_{\mathbb{C}}$ determined by their boundary values on $G / H$. Similarly, we have the "classical" Hardy spaces on $G_{1} / K_{1}$ - or the biholomorphically equivalent tube domain - formed by certain holomorphic functions on $G_{1} / K_{1}$ determined by their boundary values on the Bergman-Šilov boundary $G_{1} / P^{\prime}$. Causality of $\Phi$ assures that it can be prolonged, at least locally, to a map $\tilde{\Phi}$ from the mentioned open domain in $G_{\mathbb{C}} / H_{\mathbb{C}}$ into $G_{1} / K_{1}$. Thus $\tilde{\Phi}^{*}$ maps functions on $G_{1} / K_{1}$ to functions on the domain of definition for the Hardy space, and this can be used to construct an imbedding of the "classical" Hardy space into the new one. This program is carried out only for Cayley spaces ([ÓØ 97]) and some of the group cases $(\mathfrak{g}, \mathfrak{h}) \simeq(\tilde{\mathfrak{g}} \oplus \tilde{\mathfrak{g}}, \tilde{\mathfrak{g}})$ (KØ96, KØ97]) of our list above, and, as a main result, gives explicit formulas for the Cauchy-Szegö kernel. The general case is considered in [FÓ].

Results similar to ours were also obtained by Bertram ([Be] using Jordan algebra methods. The method used by us has the advantage that it allows one to find the $G$-orbits in the compactification by looking not only at the open cosets but using the full double coset decomposition of Matsuki. For example, for spaces of Cayley type one recovers the orbit decomposition given by Kaneyuki ([Ka 87]).

I am grateful to H. Holdgrün and G. Ólafsson for their continuing interest, and for discussions concerning the content of the present paper. I would also like to thank the referee for several suggestions that improved the exposition considerably.

\footnotetext{
${ }^{1}$ Here $p \geq 2$, whereas for the other cases $p$ and $q$ are only nonnegative with $p+q=n$.
} 


\section{BASIC STRUCTURE THEORY}

Let $\left(\mathfrak{g}_{1}, \mathfrak{k}_{1}, \theta\right)$ be an (irreducible) orthogonal symmetric Lie algebra of noncompact Hermitian type. Denote the complexification of an algebra or group by a subscript $\mathbb{C}$, for example $\mathfrak{g}_{1 \mathbb{C}}=\mathfrak{g}_{1} \otimes \mathbb{C}$. Let $G_{1 \mathbb{C}}$ be the simply connected group with Lie algebra $\mathfrak{g}_{1 \mathbb{C}}$, and let $\left(G_{1}, K_{1}, \theta\right)$ be associated to $\left(\mathfrak{g}_{1}, \mathfrak{k}_{1}, \theta\right)$ with $G_{1} \subset G_{1 \mathbb{C}}$. For simplicity we will always denote the Cartan involution $\theta$ for $G_{1}$ or $\mathfrak{g}_{1}$ and its extension to the respective complexifications by the same letter. (The same notational convention will be used without further comment for all other morphisms.) Let $\mathfrak{g}_{1}=\mathfrak{k}_{1}+\mathfrak{p}_{1}$ be the Cartan decomposition for $\theta$.

Let $\mathfrak{t} \subset \mathfrak{k}_{1}$ be a Cartan subalgebra and $\Delta\left(\mathfrak{g}_{1 \mathbb{C}}, \mathfrak{t}_{\mathbb{C}}\right)$ the corresponding root system. Choose a positive system $\Delta^{+}\left(\mathfrak{g}_{1 \mathbb{C}}, \mathfrak{t}_{\mathbb{C}}\right)$ such that the noncompact roots $\Delta^{+}\left(\mathfrak{p}_{1 \mathbb{C}}, \mathfrak{t}_{\mathbb{C}}\right)$ dominate the compact roots $\Delta^{+}\left(\mathfrak{k}_{1 \mathbb{C}}, \mathfrak{t}_{\mathbb{C}}\right)$. Recall that two roots $\alpha$ and $\beta$ are strongly orthogonal if $\alpha \neq \pm \beta$ and $\alpha \pm \beta \notin \Delta\left(\mathfrak{g}_{1 \mathbb{C}}, \mathfrak{t}_{\mathbb{C}}\right)$. Let $\left\{\gamma_{1}, \ldots, \gamma_{r}\right\} \subset \Delta^{+}\left(\mathfrak{p}_{1 \mathbb{C}}, \mathfrak{t}_{\mathbb{C}}\right)$ be a maximal system of strongly orthogonal roots. Choose $E_{ \pm j} \in \mathfrak{g}_{1 \mathbb{C}, \pm \gamma_{j}}$, where $\mathfrak{g}_{1 \mathbb{C}, \gamma}$ denotes the root space for $\gamma$, such that

$$
X_{j}:=E_{j}+E_{-j} \in \mathfrak{k}_{1}, \quad Y_{j}:=i E_{j}-i E_{-j} \in \mathfrak{p}_{1},
$$

and

$$
\left[E_{j}, E_{-j}\right]=H_{j}
$$

Here $H_{j} \in i$ is defined by

$$
\left\langle H_{j}, H\right\rangle=2 \frac{\gamma_{j}(H)}{\left\langle\gamma_{j}, \gamma_{j}\right\rangle}
$$

for all $H \in \mathfrak{t}([\mathrm{He} 78$ p. 387]). (As usual, define the pairing for $\operatorname{roots} \alpha, \beta$ by

$$
\langle\alpha, \beta\rangle=\left\langle H_{\alpha}, H_{\beta}\right\rangle=B\left(H_{\alpha}, H_{\beta}\right),
$$

where $B$ is the Killing form of $\mathfrak{g}_{1}$ and $H_{\alpha} \in \mathfrak{t}_{\mathbb{C}}$ the vector dual to $\alpha$ with respect to $B$.) It is known that $\mathfrak{a}=\sum \mathbb{R} X_{j}$ is maximal abelian in $\mathfrak{p}_{1}$.

Define the (full) Cayley transform by

$$
c:=\prod_{j=1}^{r} c_{j}, \quad \text { where } c_{j}=\exp \frac{\pi}{4} i Y_{j} .
$$

By the strong orthogonality of the $\gamma_{j}$ 's, an $\mathfrak{s l}(2)$-calculation shows $\operatorname{Ad}(c) i H_{j}=X_{j}$ and $\operatorname{Ad}(c) i \mathfrak{t}^{-}=\mathfrak{a}$, where $\mathfrak{t}^{-}:=\sum \mathbb{R} i H_{j} \subset \mathfrak{t}$. By abuse of notation, let $\gamma_{j}$ also denote the restriction of $\gamma_{j}$ to $\mathfrak{t}^{-}$. The restricted root system is then given by the theorem of Moore.

Theorem 2.1 (Moore, [He 78, p. 528]). For the restricted root system $\Delta\left(\mathfrak{g}_{1 \mathbb{C}}, \mathfrak{t}_{\mathbb{C}}^{-}\right)$ one of the following cases occurs:

(1) $\Delta\left(\mathfrak{g}_{1 \mathbb{C}}, \mathfrak{t}_{\mathbb{C}}^{-}\right)=\left\{ \pm \gamma_{j} \mid 1 \leq j \leq r\right\} \cup\left\{ \pm \frac{1}{2} \gamma_{i} \pm \frac{1}{2} \gamma_{j} \mid 1 \leq i<j \leq r\right\}$; or

(2) $\Delta\left(\mathfrak{g}_{1 \mathbb{C}}, \mathfrak{t}_{\mathbb{C}}^{-}\right)=\left\{ \pm \frac{1}{2} \gamma_{j} \mid 1 \leq j \leq r\right\} \cup\left\{ \pm \gamma_{j} \mid 1 \leq j \leq r\right\} \cup\left\{ \pm \frac{1}{2} \gamma_{i} \pm \frac{1}{2} \gamma_{j} \mid 1 \leq\right.$ $i<j \leq r\}$.

Using the Cayley transform, the structure of the root system $\Sigma(\mathfrak{a}):=\Delta\left(\mathfrak{g}_{1}, \mathfrak{a}\right)$ can be immediately derived from this theorem.

For example, assume for the moment $\left(G_{1}, K_{1}, \theta\right)$ or $\left(\mathfrak{g}_{1}, \mathfrak{k}_{1}, \theta\right)$ to be Hermitian symmetric of tube type, i.e. $G_{1} / K_{1}$ is biholomorpically equivalent to a bounded symmetric domain of tube type. For this case the restricted root system is of 
type $\mathfrak{c}_{r}$ ( $\mathrm{KW} 65$ Prop. 3.12]) such that in the theorem above the root system is given by the first case. Therefore we have

$$
\Sigma(\mathfrak{a})=\left\{ \pm \gamma_{j}^{c} \mid 1 \leq j \leq r\right\} \cup\left\{ \pm \frac{1}{2} \gamma_{i}^{c} \pm \frac{1}{2} \gamma_{j}^{c} \mid 1 \leq i<j \leq r\right\}
$$

where $\gamma_{j}^{c}:=\gamma_{j} \circ \operatorname{Ad}\left(c^{-1}\right)$ is the Cayley transformed root. Furthermore, $\gamma_{i}^{c}\left(X_{j}\right)=$ $2 \delta_{i j}$, such that the co-roots to the $\gamma_{j}^{c}$ 's are up to normalization the $X_{j}$ 's. Since the Weyl group $W(\mathfrak{a})$, which we consider to operate on the roots as well as on the $X_{j}$ 's, is determined by the root system, we get also

Corollary 2.2. For $\left(\mathfrak{g}_{1}, \mathfrak{k}_{1}, \theta\right)$ Hermitian symmetric of tube type we have $W(\mathfrak{a}) \simeq$ $(\mathbb{Z} / 2 \mathbb{Z})^{r} \rtimes S_{r}$, where the permutations in $S_{r}$ operate by permuting indices and $(\mathbb{Z} / 2 \mathbb{Z})^{r} \simeq\left\{\left(\epsilon_{1}, \ldots, \epsilon_{r}\right) \mid \epsilon_{j} \in\{ \pm 1\}\right\}$ by sign changes on $\left(X_{1}, \ldots, X_{r}\right)$.

With $\mathfrak{p}_{1}^{+}:=\sum_{\gamma \in \Delta^{+}\left(\mathfrak{p}_{1 \mathbb{C}}, \mathfrak{t}_{\mathbb{C}}\right)} \mathfrak{g}_{1 \mathbb{C}, \gamma}, \mathfrak{p}_{1}^{-}:=\sum_{\gamma \in \Delta^{+}\left(\mathfrak{p}_{1 \mathbb{C}}, \mathfrak{t}_{\mathbb{C}}\right)} \mathfrak{g}_{1 \mathbb{C},-\gamma}$, and

$$
\mathfrak{g}_{1 \mathbb{C}}=\mathfrak{p}_{1}^{+}+\mathfrak{k}_{1 \mathbb{C}}+\mathfrak{p}_{1}^{-}
$$

the usual decomposition for a Hermitian symmetric algebra ([He 78, Ch. VIII]), $P_{1}^{ \pm}$ and $K_{1 \mathbb{C}}$ the corresponding analytic subgroups, let $x_{0}:=e K_{1 \mathbb{C}} P_{1}^{-} \in G_{1 \mathbb{C}} / K_{1 \mathbb{C}} P_{1}^{-}$ $=: M^{*}$ be the coset of the identity in the Borel realization of the Hermitian symmetric space $G_{1} / K_{1}$. The Bergman-Šilov boundary $\breve{S}_{1}$ of $G_{1} / K_{1}$ is the closed boundary orbit $G_{1}\left(c x_{0}\right)$ ( $\left.\mathrm{KW} 65\right)$. With an $\mathfrak{s l}(2)$-calculation,

$$
c_{j}=\exp \left(-E_{j}\right) \exp \left(\log \sqrt{2} H_{j}\right) \exp \left(E_{-j}\right) .
$$

Thus in the Harish-Chandra realization of $G_{1} / H_{1}$ in $\mathfrak{p}_{1}^{+}$, the Bergman-Šilov boundary is given as the $G_{1}$-orbit through $-\sum E_{j}$. This boundary can also be described as the $G_{1}$-orbit of $c^{-1} x_{0}$ or $\sum E_{j}$. Indeed, $c^{-1} x_{0}=\theta(c)^{-1} x_{0}=\exp \left(\sum E_{j}\right) \cdot x_{0}$. This is an element of the Bergman-Šilov boundary, since the exponential of the

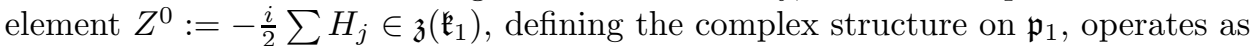
complex rotations $\left(z \mapsto e^{i \lambda} z\right)$ on $\mathfrak{p}_{1}^{+}$.

For $X^{0}:=\sum_{j=1}^{r} X_{j}$ the eigenvalues of ad $X^{0}$ are from $\{0, \pm 1, \pm 2\}$. We define $\mathfrak{p}_{\mathbb{C}}^{\prime}$ to be the sum of the eigenspaces of ad $X^{0}$ in $\mathfrak{g}_{1 \mathbb{C}}$ with nonpositive eigenvalues, and $\mathfrak{q}_{1}^{+}\left(\mathfrak{q}_{1}^{-}\right)$to be the sum of the eigenspaces in $\mathfrak{g}_{1}$ with positive (respectively negative) eigenvalues. Let $P_{\mathbb{C}}^{\prime}$ respectively $Q_{1}^{+}\left(Q_{1}^{-}\right)$be the corresponding analytic subgroups in $G_{1 \mathbb{C}}$ respectively $G_{1}$.

Theorem 2.3 ([Wo 72, I.6]). The stabilizer of the boundary point $c K_{1 \mathbb{C}} P_{1}^{-}$of $G_{1} / K_{1}$ is given by $P^{\prime}:=P_{\mathbb{C}}^{\prime} \cap G_{1}$. Furthermore, if $G_{1}$ is simple, then $P^{\prime}$ is a maximal parabolic subgroup of $G_{1}$.

The theorem shows that $\check{S}_{1} \simeq G_{1} / P^{\prime}$, and we will call this homogeneous space the Bergman-Šilov boundary, too.

We also note for later use that for the parabolic subgroup $P^{\prime}$ we have the Langlands decomposition

$$
P^{\prime} \simeq M^{\prime} \times A^{\prime} \times Q_{1}^{-},
$$

where $A^{\prime}:=\exp \mathbb{R} X^{0}$ and $M^{\prime} A^{\prime}=Z_{G_{1}}\left(X^{0}\right)$. 


\section{Causal structures for Cayley spaces AND THE BERGMAN-ŠILOV BOUNDARY}

We will from now on always assume $\left(\mathfrak{g}_{1}, \mathfrak{k}_{1}, \theta\right)$ to be of tube type. Then ad $X^{0}$ has eigenvalues 0 and \pm 2 . The inner automorphism

$$
\eta:=\operatorname{Ad}\left(\exp \frac{\pi}{2} i X^{0}\right)
$$

is therefore involutive, and we get $H_{1}:=G_{1}^{\eta}=Z_{G_{1}}\left(X^{0}\right)$ and $\theta \eta=\eta \theta$. The triple $\left(G_{1}, H_{1}, \eta\right)$ defines an (irreducible) symmetric space of Cayley type, and all Cayley spaces (up to covering) are obtained in this way (HÓ 96 section 2.6]).

Let us recall the definition of the compactly causal structure on $G_{1} / H_{1}$, cf. HÓ 96 section 2.6]. Decomposing $Y^{0}:=\sum_{j=1}^{r} Y_{j}$ in $( \pm 2)$-eigenvectors $Y_{ \pm}$of ad $X^{0}$ - it is immediate to verify that $Y_{ \pm}=\frac{1}{2} \sum_{j=1}^{r}\left(Y_{j} \mp i H_{j}\right)$ - we define $C_{ \pm}:=\overline{\operatorname{conv} \operatorname{Ad}\left(H_{1}\right)_{0} \mathbb{R}^{+} Y_{ \pm}}$, which are $H_{1}$-invariant cones. Then

$$
C_{k}:=C_{+}-C_{-} \subset \mathfrak{q}_{1}^{+} \oplus \mathfrak{q}_{1}^{-}=: \mathfrak{q}_{1}
$$

is a regular $H_{1}$-invariant cone. We have the canonical identification of $\mathfrak{q}_{1}$, which is the (-1)-eigenspace of $\eta$, with the tangent space $T_{e H_{1}} G_{1} / H_{1}$, and, using this identification, $C_{k}$ defines the compactly causal structure of $G_{1} / H_{1}$.

We also want to endow the Bergman-Šilov boundary with a $G_{1}$-equivariant causal structure (cf. [Ka 89]). With $\breve{S}_{1}=G_{1}\left(c^{-1} x_{0}\right)$ we define the Cayley transform $\breve{S}_{1}^{c}=c G_{1}\left(c^{-1} x_{0}\right)=\left(\operatorname{Ad}(c) G_{1}\right) x_{0}$. Note that the left multiplication $L_{c}$ on $M^{*}$ defines a diffeomorphism of $\check{S}_{1}^{c}$ onto $\check{S}_{1}$ if $G_{1} / K_{1}$ is of tube type. Indeed, to be of tube type is equivalent to $\operatorname{Ad}\left(c^{2}\right) \mathfrak{k}_{1}=\mathfrak{k}_{1}$ ( $\underline{\mathrm{K} W 65}$, Prop. 4.4]), which implies $\operatorname{Ad}\left(c^{2}\right) \mathfrak{g}_{1}=\mathfrak{g}_{1}$, and then we have $L_{c} \check{S}_{1}^{c}=\left(\operatorname{Ad}\left(c^{2}\right) G_{1}\right)\left(c x_{0}\right)=G_{1}\left(c x_{0}\right)=\check{S}_{1}$.

Let us identify the tangent space $T_{x_{0}} M^{*}$ with $\mathfrak{p}_{1}^{+}$by use of the differential of the map $\xi: \mathfrak{p}_{1}^{+} \rightarrow M^{*}, X \mapsto \exp X \cdot x_{0}$. Thereby the subspace $T_{x_{0}} \check{S}_{1}^{c}$ corresponds to $\mathfrak{s}_{1}^{+}:=\mathfrak{p}_{1}^{+} \cap \operatorname{Ad}(c) \mathfrak{g}_{1}$, and this space can be given the structure of a Euclidean or formally real Jordan algebra ( $\mathrm{KW} 65$, section 6$])$. The cone defining the causal structure of $\check{S}_{1}^{c}$ is then given by the identity component of the set of all squares in $\mathfrak{s}_{1}^{+}$ ( Ka 89]), or equivalently the closure of the $K_{1}^{c}$-orbit of $E:=-i \xi^{-1}\left(c x_{0}\right)=i \sum E_{j}$, with $K_{1}^{c}:=K_{1 \mathbb{C}} \cap \operatorname{Ad}(c) G_{1}$. We define the causal structure of $\check{S}_{1}$ now by claiming that $L_{c}$ is a causal diffeomorphism.

Let us describe this causal structure more explicitly. First of all, by use of the differential of $\xi^{c}: \operatorname{Ad}(c) \mathfrak{p}_{1}^{+} \rightarrow M^{*}, \operatorname{Ad}(c) X \mapsto \exp (\operatorname{Ad}(c) X) c x_{0}$, we identify the spaces $\operatorname{Ad}(c) \mathfrak{p}_{1}^{+}$and $T_{c x_{0}} M^{*}$. For spaces of tube type $\operatorname{Ad}(c)\left(i Z^{0}\right)=\frac{1}{2} X^{0}$, and correspondingly $\operatorname{Ad}(c) \mathfrak{p}_{1}^{+}$is the $(+2)$-eigenspace $\mathfrak{q}_{1 \mathbb{C}}^{+}$of ad $X^{0}$ in $\mathfrak{g}_{1 \mathbb{C}}$. We get

$$
\operatorname{Ad}(c) \mathfrak{s}_{1}^{+}=\operatorname{Ad}(c) \mathfrak{p}_{1}^{+} \cap \operatorname{Ad}\left(c^{2}\right) \mathfrak{g}_{1}=\operatorname{Ad}(c) \mathfrak{p}_{1}^{+} \cap \mathfrak{g}_{1}=\mathfrak{q}_{1}^{+}
$$

for the tangent space $T_{c x_{0}} \breve{S}_{1}$ (with our identifications). Denote by $H_{1 \mathbb{C}}$ the analytic subgroup corresponding to $\mathfrak{h}_{1 \mathbb{C}}:=\mathfrak{z}_{\mathfrak{g}_{1 \mathbb{C}}}\left(X^{0}\right)$; then

$$
H_{1}=H_{1 \mathbb{C}} \cap G_{1}=\operatorname{Ad}(c) K_{1 \mathbb{C}} \cap G_{1}=\operatorname{Ad}(c) K_{1}^{c} .
$$

Together with $\operatorname{Ad}(c) E=Y_{+}$, our above definition now shows that the defining cone in $\mathfrak{q}_{1}^{+}$is the closure of the $H_{1}$-orbit of $Y_{+}$, i.e. $C_{+}=\overline{\operatorname{Ad}\left(H_{1}\right) Y_{+}}$.

Remark. Alternatively, one can proceed as follows, cf. [Ka 89]. Since the cone $C_{+}=\overline{\operatorname{conv} \operatorname{Ad}\left(H_{1}\right)_{0} \mathbb{R}^{+} Y_{+}}$is regular and $H_{1}$-invariant in $\mathfrak{q}_{1}^{+}$, it is easy to see that this cone is invariant under the linear isotropy representation of $P^{\prime}$ in $T_{e P^{\prime}} G_{1} / P^{\prime}$. 
It therefore defines a $G_{1}$-equivariant causal structure on $G_{1} / P^{\prime}$, unique up to sign change.

Since the tangent map of the projection

$$
\Phi: G_{1} / H_{1} \rightarrow G_{1} / P^{\prime}
$$

at the identity coset is given, using the identifications introduced above, by the projection of $\mathfrak{q}_{1}=\mathfrak{q}_{1}^{+} \oplus \mathfrak{q}_{1}^{-}$onto $\mathfrak{q}_{1}^{+}$, and the fact both causal structures are $G_{1}$-equivariant, this projection of the Cayley space $G_{1} / H_{1}$ on the Bergman-Šilov boundary is causal.

Remark. The projection is also causal if we consider the Cayley space with its noncompactly causal structure defined by $C_{p}:=C_{+}+C_{-}$.

Later on we will consider subspaces $G / H \subset G_{1} / H_{1}$. The question arises: When is $G / H$ a causal subspace, i.e., when does $G / H$ have a causal structure such that $G / H \hookrightarrow G_{1} / H_{1}$ is causal? If $G=G_{1}^{\sigma}$ is the fixed point group of an involution $\sigma$, the following lemma gives a simple criterion.

Lemma 3.1. Let $\sigma$ be an involution commuting with $\eta$ and $\theta$. Let $C \subset \mathfrak{q}_{1}$ be a convex generating $\sigma$-invariant cone. Let $\mathfrak{q}_{1 \sigma}$ be the $(-1)$-eigenspace of $\sigma$ and $\mathfrak{q}_{1}=\mathfrak{q}_{1}^{\sigma} \oplus \mathfrak{q}_{1} \cap \mathfrak{q}_{1 \sigma}$ the decomposition in $( \pm 1)$-eigenspaces of $\sigma$. Then $C \cap \mathfrak{q}_{1}^{\sigma}=\operatorname{pr}_{\mathfrak{q}_{1}^{\sigma}} C$, and this cone is generating in $\mathfrak{q}_{1}^{\sigma}$.

Proof. For $v \in \operatorname{pr}_{\mathfrak{q}_{1}^{\sigma}} C$ there exists $w \in \mathfrak{q}_{1} \cap \mathfrak{q}_{1 \sigma}$ such that $v+w \in C$. By $\sigma$-invariance $v-w \in C$, and then $v=\frac{1}{2}(v+w)+\frac{1}{2}(v-w) \in C$, which proves that $\operatorname{pr}_{\mathfrak{q}_{1}^{\sigma}} C \subset C \cap \mathfrak{q}_{1}^{\sigma}$. The other inclusion being trivial, we finally have

$$
\mathfrak{q}_{1}^{\sigma}=\operatorname{pr}_{\mathfrak{q}_{1}^{\sigma}}(C-C)=\operatorname{pr}_{\mathfrak{q}_{1}^{\sigma}} C-\operatorname{pr}_{\mathfrak{q}_{1}^{\sigma}} C .
$$

\section{Double coset Decompositions}

In this section we recall some results of Matsuki on the $\left(G, P^{\prime}\right)$-cosets in $G_{1}$, where $G$ is an open subgroup of the fixed point group of an involution and $P^{\prime}$ a parabolic subgroup.

If we look at minimal parabolic subgroups, these can be parametrized in the usual manner by assigning a maximal abelian subspace $\mathfrak{a}$ of $\mathfrak{p}_{1}$ and a system of positive roots $\Sigma^{+} \subset \Sigma(\mathfrak{a})$, with $\Sigma(\mathfrak{a})$ the root system of $\mathfrak{g}_{1}$ with respect to $\mathfrak{a}$. We indicate this by writing $P\left(\mathfrak{a}, \Sigma^{+}\right)$, and $\mathfrak{p}\left(\mathfrak{a}, \Sigma^{+}\right)$for the algebra. For a subset $\mathfrak{r}$ of $\mathfrak{a}$ we define a subset of (positive) roots by $\Sigma^{(+)}(\mathfrak{r}):=\left\{\gamma \in \Sigma^{(+)} \mid H_{\gamma} \in \mathfrak{r}\right\}$, where $H_{\gamma} \in \mathfrak{a}$ is the co-root to $\gamma$ defined by $B\left(H_{\gamma}, H\right)=\gamma(H)$ for all $H \in \mathfrak{a}$. Since all minimal parabolic subgroups are conjugate and self-normalizing, we can identify the factor space $G_{1} / P$ with the set of all minimal parabolic subalgebras of $\mathfrak{g}_{1}$.

Let $\left(G_{1}, G, \sigma\right)$ be a symmetric triple, i.e. $\left(G_{1}^{\sigma}\right)_{0} \subset G \subset G_{1}^{\sigma}$, and assume $\sigma$ to commute with $\theta$. Let $\mathfrak{g}_{1}=\mathfrak{g}+\mathfrak{q}_{1 \sigma}$ be the decomposition into eigenspaces of $\sigma$, and $\mathfrak{a}$ a $\sigma$-stable maximal abelian subspace of $\mathfrak{p}_{1}$. We define two subgroups of the Weyl group $W(\mathfrak{a})=N_{K_{1}}(\mathfrak{a}) / Z_{K_{1}}(\mathfrak{a})$ by

$$
W_{\sigma}(\mathfrak{a}):=\{w \in W(\mathfrak{a}) \mid w(\mathfrak{a} \cap \mathfrak{g})=\mathfrak{a} \cap \mathfrak{g}\}
$$

and

$$
W\left(\mathfrak{a}, K_{1} \cap G\right):=N_{K_{1} \cap G}(\mathfrak{a}) / Z_{K_{1} \cap G}(\mathfrak{a}) \subset W_{\sigma}(\mathfrak{a}) .
$$


Proposition 4.1 (Ma 79 Prop. 1]). A minimal parabolic subalgebra $\mathfrak{p}\left(\mathfrak{a}, \Sigma^{+}\right)$, which is identified with a point of $G_{1} / P$, is contained in an open $G$-orbit if and only if the following two conditions are satisfied:

(i) $\mathfrak{a} \cap \mathfrak{q}_{1 \sigma}$ is maximal abelian in $\mathfrak{p}_{1} \cap \mathfrak{q}_{1 \sigma}$, and

(ii) $\Sigma^{+}$is $\sigma \theta$-compatible, i.e. $\gamma \in \Sigma^{+} \backslash \Sigma^{+}(\mathfrak{a} \cap \mathfrak{g}) \Rightarrow \sigma \theta(\gamma) \in \Sigma^{+} \backslash \Sigma^{+}(\mathfrak{a} \cap \mathfrak{g})$.

The number of open orbits is $\left|W_{\sigma}(\mathfrak{a})\right| /\left|W\left(\mathfrak{a}, K_{1} \cap G\right)\right|$.

Remark. Condition (i) determines a up to $\operatorname{Ad}\left(K_{1} \cap G\right)_{0}$-conjugacy.

Assuming $P^{\prime}$ to be an arbitrary parabolic subgroup, we want to determine the double cosets $G g P\left(\mathfrak{a}, \Sigma^{+}\right)$within $G P^{\prime}$. Let therefore $\mathfrak{p}^{\prime}=\mathfrak{m}^{\prime}+\mathfrak{a}^{\prime}+\mathfrak{n}^{\prime}$ be the Langlands decomposition of $\mathfrak{p}^{\prime}$ such that $\mathfrak{a}^{\prime} \subset \mathfrak{a}$. Define

$$
\mathfrak{a}_{+}^{\prime}:=\left\{X \in \mathfrak{a}^{\prime} \mid \gamma(X)>0 \text { for all } \gamma \text { with } \mathfrak{g}_{1, \gamma} \subset \mathfrak{n}^{\prime}\right\},
$$

where $\mathfrak{g}_{1, \gamma}$ is the root space for $\gamma \in \Sigma(\mathfrak{a})$.

Theorem 4.2 ([Ma 82]). Every minimal parabolic subalgebra of $\mathfrak{g}_{1}$ contained in $\mathfrak{p}^{\prime}$ is $G \cap P^{\prime}$-conjugate to a minimal parabolic subalgebra $\mathfrak{p}\left(\mathfrak{a}_{1}, \Sigma\left(\mathfrak{a}_{1}\right)^{+}\right)$, where $\mathfrak{a}_{1}$ is a $\sigma$-stable maximal abelian subspace of $\mathfrak{p}_{1}$ such that $\mathfrak{a}_{1} \supset \mathfrak{a}^{\prime}$ and $\Sigma\left(\mathfrak{a}_{1}\right)^{+}$satisfies $\left\langle\Sigma\left(\mathfrak{a}_{1}\right)^{+}, \mathfrak{a}_{+}^{\prime}\right\rangle \subset \mathbb{R}^{+}$.

\section{Causal compactifications}

We are now ready to state and prove our main results. If not otherwise stated, our notation will be as introduced in the previous sections.

Theorem 5.1. Let $\left(G_{1}, H_{1}, \eta\right)$ be an irreducible causal symmetric space of Cayley type with $G_{1} \subset G_{1 \mathbb{C}}, G_{1 \mathbb{C}}$ the simply connected Lie group corresponding to $\mathfrak{g}_{1 \mathbb{C}}$. Let $\sigma$ be an involution commuting with $\eta$ and $\theta$. Assume one of the following cases holds:

(i) $\sum \mathbb{R} X_{j}$ and $\sum \mathbb{R} Y_{j}$ are maximal abelian in $\mathfrak{p}_{1} \cap \mathfrak{q}_{1 \sigma}$, where $\mathfrak{q}_{1 \sigma}$ is the $(-1)$ eigenspace of the involution $\sigma$.

(ii) We have $r=2 r^{\prime}$, and, with $X_{j^{\prime}}^{\prime}:=X_{j^{\prime}}+X_{j^{\prime}+r^{\prime}}, Y_{j^{\prime}}^{\prime}:=Y_{j^{\prime}}+Y_{j^{\prime}+r^{\prime}}$, for $j^{\prime}=1, \ldots, r^{\prime}$, the spaces $\sum \mathbb{R} X_{j^{\prime}}^{\prime}$ and $\sum \mathbb{R} Y_{j^{\prime}}^{\prime}$ are maximal abelian in $\mathfrak{p}_{1} \cap \mathfrak{q}_{1 \sigma}$.

Then $(G, H, \tau):=\left(\left(G_{1}^{\sigma}\right)_{0}, H_{1} \cap\left(G_{1}^{\sigma}\right)_{0}, \eta \mid\left(G_{1}^{\sigma}\right)_{0}\right)$ is a weak compactly causal subspace of $\left(G_{1}, H_{1}, \eta\right)$, and $\Phi: G / H \rightarrow G_{1} / P^{\prime}$ is a causal compactification.

Proof. For the eigenspaces of ad $X^{0}$ we have $\sigma\left(\mathfrak{q}_{1}^{-}\right)=\mathfrak{q}_{1}^{+}$, since $X^{0} \in \sum \mathbb{R} X_{j}$ and $X^{0} \in \sum \mathbb{R} X_{j^{\prime}}^{\prime}$. Using the Langlands decomposition of $P^{\prime}$, this implies on the group level $Q_{1}^{-} \cap G=\{e\}$ and $G \cap P^{\prime}=G \cap H_{1}=H$, i.e. $\Phi$ is injective. The subspace $T_{e H} G / H \subset T_{e H_{1}} G_{1} / H_{1}$ corresponds with our identifications to $\left\{X+\sigma X \mid X \in \mathfrak{q}_{1}^{+}\right\}$, and the restriction of the tangent map of $\Phi$ is given by $T \Phi(X+\sigma X)=X$. Therefore, by $G$-equivariance, $\Phi$ is a submersion onto an open image.

Let us show the density of the image. From (i) $\mathfrak{a}:=\sum \mathbb{R} X_{j}$ is a maximal abelian $\sigma$-invariant subspace of $\mathfrak{p}_{1}$, and the first condition of Proposition 4.1 is fulfilled. Assume $\Sigma(\mathfrak{a})^{+}$to be $\sigma \theta$-compatible; then all other positive systems are conjugates $w\left(\Sigma(\mathfrak{a})^{+}\right)$, with $w \in W(\mathfrak{a})$. The system $w\left(\Sigma(\mathfrak{a})^{+}\right)$is again $\sigma \theta$-compatible if and only if $w \in W_{\sigma}(\mathfrak{a})$. Indeed, by Theorem 2.1 and the following discussion, we have $\Sigma(\mathfrak{a})=\left\{ \pm \frac{1}{2}\left(\gamma_{s}^{c}+\gamma_{t}^{c}\right), \pm \frac{1}{2}\left(\gamma_{u}^{c}-\gamma_{v}^{c}\right) \mid 1 \leq s, t, u, v \leq r, u \neq v\right\}$, and $X_{j}$ is up to normalization the co-root to $\gamma_{j}^{c}$. Therefore, identifying $\mathfrak{a}$ and its dual 
$\mathfrak{a}^{*}, \mathfrak{a} \cap \mathfrak{g}$ is spanned by $\Sigma(\mathfrak{a} \cap \mathfrak{g})^{+}$. By Proposition 4.1 the $\left(G, P\left(\mathfrak{a}, \Sigma^{+}\right)\right)$-double cosets corresponding to open orbits in $G_{1} / P\left(\mathfrak{a}, \Sigma^{+}\right)$are then given by $G w_{v} P\left(\mathfrak{a}, \Sigma^{+}\right)$, where $\Sigma^{+}$is an arbitrary $\sigma \theta$-compatible positive system and $w_{v}$ a representative for $v \in W\left(\mathfrak{a}, K_{1} \cap G\right) \backslash W_{\sigma}(\mathfrak{a})$.

It is now enough to show that all these open double cosets lie in $G P^{\prime}$, and we use Theorem 4.2 to do this. With $\mathfrak{a}^{\prime}=\mathbb{R} X^{0}$ we have $\mathfrak{a}_{+}^{\prime}=-\mathbb{R}^{+} X^{0}$, as $\mathfrak{q}_{1}^{-}$is the $(-2)$-eigenspace of ad $X^{0}$. Let $\Sigma(\mathfrak{a})^{+}$fulfill the condition of Theorem 4.2, i.e. $\left\langle\Sigma(\mathfrak{a})^{+}, X^{0}\right\rangle \leq 0$; then $w\left(\Sigma(\mathfrak{a})^{+}\right)$obeys the same condition if and only if

$$
\left\langle w\left(\Sigma(\mathfrak{a})^{+}\right), \mathfrak{a}_{+}^{\prime}\right\rangle=-\mathbb{R}^{+}\left\langle\Sigma(\mathfrak{a})^{+}, w^{-1}\left(X^{0}\right)\right\rangle \geq 0
$$

or equivalently $w\left(X^{0}\right)=X^{0}$. This shows that the open orbits lying in $G P^{\prime}$ are parametrized by $W(\mathfrak{a})_{\mathfrak{m}^{\prime}} \cap W\left(\mathfrak{a}, K_{1} \cap G\right) \backslash W(\mathfrak{a})_{\mathfrak{m}^{\prime}}$, where

$$
W(\mathfrak{a})_{\mathfrak{m}^{\prime}}:=N_{K_{1} \cap M^{\prime}}(\mathfrak{a}) / Z_{K_{1} \cap M^{\prime}}(\mathfrak{a})
$$

is the subgroup of the Weyl group leaving $X^{0}$ fixed. With the commutative diagram

$$
\begin{aligned}
W(\mathfrak{a})_{\mathfrak{m}^{\prime}} & \cap W_{\sigma}(\mathfrak{a}) \rightarrow W_{\sigma}(\mathfrak{a}) \rightarrow W\left(\mathfrak{a}, K_{1} \cap G\right) \backslash W_{\sigma}(\mathfrak{a}) \\
& \downarrow \\
W(\mathfrak{a})_{\mathfrak{m}^{\prime}} & \cap W\left(\mathfrak{a}, K_{1} \cap G\right) \backslash W(\mathfrak{a})_{\mathfrak{m}^{\prime}} \cap W_{\sigma}(\mathfrak{a}),
\end{aligned}
$$

it remains to show the surjectivity of $\varphi$. But this is a consequence of Corollary 2.2 Indeed, since the permutations preserve $X^{0}=\sum_{j=1}^{r} X_{j}$, for our case (i) it is enough to show that the subgroup isomorphic to $(\mathbb{Z} / 2 \mathbb{Z})^{r} \times\{$ id $\}$ lies within $W\left(\mathfrak{a}, K_{1} \cap G\right)$. But with $X_{j}, Y_{j} \in \mathfrak{p}_{1} \cap \mathfrak{q}_{1 \sigma}$ and therefore $\left[X_{j}, Y_{j}\right]=-2 i H_{j} \in \mathfrak{k}_{1} \cap \mathfrak{g}$ this is a direct consequence of

$$
\operatorname{Ad}\left(\exp \frac{\pi}{2} i H_{j}\right)\left(E_{ \pm k}\right)=\left\{\begin{aligned}
E_{ \pm k} & \text { for } k \neq j \\
-E_{ \pm k} & \text { for } k=j
\end{aligned}\right.
$$

For case (ii) we similarly have to prove that the "sign changes" in $W_{\sigma}(\mathfrak{a})$ are elements of $W\left(\mathfrak{a}, K_{1} \cap G\right)$. These sign changes are generated by the reflections which map $X_{j^{\prime}}^{\prime}=X_{j^{\prime}}+X_{j^{\prime}+r^{\prime}}$ to its negative and leave all vectors orthogonal to this one fixed. Here $X_{j^{\prime}}^{\prime}, Y_{j^{\prime}}^{\prime} \in \mathfrak{p}_{1} \cap \mathfrak{q}_{1 \sigma}$. Thus we have $\left[X_{j^{\prime}}^{\prime}, Y_{j^{\prime}}^{\prime}\right] \in \mathfrak{k}_{1} \cap \mathfrak{g}_{1}$, and the needed result follows again from (11).

Knowing that $\Phi$ is a $G$-equivariant diffeomorphism onto an open and dense subset, it remains to prove the claims concerning causality. We consider first the case (i). With $\sigma\left(Y_{j}\right)=-Y_{j}$ or $\sigma\left(i H_{j}\right)=i H_{j}$ we get $\sigma\left(Y_{+}\right)=-Y_{-}$by the definition of $Y_{ \pm}$, cf. section 3 . This implies $\sigma\left(C_{ \pm}\right)=-C_{\bar{\mp}}$ and the $\sigma$-invariance of $C_{k}$. The cone $C_{k}$ being regular $H_{1}$-invariant, we see by Lemma 3.1 that $C_{k} \cap \mathfrak{g} \cap \mathfrak{q}_{1}$ is an $H$-invariant regular cone in $\mathfrak{g} \cap \mathfrak{q}_{1}$. This cone has nontrivial intersection with $\mathfrak{k}_{1} \cap \mathfrak{g}$, as $Y_{+}-Y_{-}=2 Z^{0} \in \mathfrak{k}_{1} \cap \mathfrak{g}$, i.e. $G / H$ is weak compactly causal. Case (ii) is treated in the same way, with the obvious modifications. Finally, since we proved $\Phi: G_{1} / H_{1} \rightarrow G_{1} / P^{\prime}$ to be causal, it is clear that the restriction of this map to the causal subspace $G / H$ is causal, too.

Remark. 1. Note that $C_{p}=C_{+}+C_{-}$will be mapped by $\sigma$ to $-C_{p}$, and therefore $G / H$ is not a noncompactly causal subspace of the Cayley space $G_{1} / H_{1}$. In turn this shows that $G_{a} / H_{1} \cap G_{a}$, with $G_{a}:=\left(G_{1}^{\sigma \theta}\right)_{0}$ the associated group to $G$, is a Cayley subspace.

2. The maximality condition for case (ii) is equivalent to the fact that $G_{a}$ has real rank $r^{\prime}$. 
In general, for $G_{1 \mathbb{C}}$ not simply connected, some of the inclusions $\left(G_{1}\right)_{0} \subset\{g \in$ $\left.G_{1 \mathbb{C}} \mid \bar{g}=g\right\}=: G_{1}$, where $\bar{g}$ denotes conjugation with respect to the real form $\mathfrak{g}_{1}$, and $\left(G_{1}^{\eta}\right)_{0} \subset Z_{G_{1}}\left(X^{0}\right) \subset G_{1}^{\eta}=H_{1}$ may be proper. For the first case, since we are finally interested in quotients like $G_{1} / H_{1}$ and $G_{1} / P^{\prime}$, it does not matter if we work with the group of all "real" matrices $G_{1}$ or its identity component. For the second case we have to replace $H_{(1)}$ by $Z_{G_{(1)}}\left(X^{0}\right)$ everywhere in our statements, and all arguments will go through without any change. For the classical groups both complications will only occur for $G_{1}=S O(2, n)$.

Example 5.2 (Causal compactification of $S O(2, n) / S O(1, n)$ ). We start with

$$
G_{1}=\left\{\left(\begin{array}{ll}
A & B \\
C & D
\end{array}\right) \in S L(n+3, \mathbb{R}) \mid \begin{array}{l}
{ }^{t} A A-{ }^{t} C C=I, \quad{ }^{t} A B={ }^{t} C D \\
{ }^{t} D D-{ }^{t} B B=I
\end{array}\right\},
$$

i.e. $G_{1}=S O(2, n+1)$, and $\theta(g)={ }^{t} g^{-1}$ the usual Cartan involution. We assume $n \geq 2$, since $\mathfrak{s o}(2,1) \simeq \mathfrak{s u}(1,1)$ and $\mathfrak{s o}(2,2) \simeq \mathfrak{s u}(1,1) \times \mathfrak{s u}(1,1)$, which thus can be treated differently. The real rank is then two, and $G_{1 \mathbb{C}}=S O(n+3, \mathbb{C})$ is not simply connected. We choose

$$
E_{1}=\frac{1}{2}\left(\begin{array}{cccc}
0 & \ldots & -i & -1 \\
\vdots & & -1 & i \\
-i & -1 & & \vdots \\
-1 & i & \ldots & 0
\end{array}\right), \quad E_{-1}=\frac{1}{2}\left(\begin{array}{cccc}
0 & \ldots & i & -1 \\
\vdots & & -1 & -i \\
i & -1 & & \vdots \\
-1 & -i & \ldots & 0
\end{array}\right)
$$

together with

$$
E_{2}=\frac{1}{2}\left(\begin{array}{cccc}
0 & \ldots & i & -1 \\
\vdots & & 1 & i \\
i & 1 & & \vdots \\
-1 & i & \ldots & 0
\end{array}\right), \quad E_{-2}=\frac{1}{2}\left(\begin{array}{cccc}
0 & \ldots & -i & -1 \\
\vdots & & 1 & -i \\
-i & 1 & & \vdots \\
-1 & -i & \ldots & 0
\end{array}\right) .
$$

The involution $\eta=\operatorname{Ad}\left(\exp i \frac{\pi}{2} X^{0}\right)$ has the fixed point group

$$
H_{1}=\left\{\left(\begin{array}{cccc}
a_{1} & 0 & 0 & b_{2} \\
0 & a_{4} & B_{3} & 0 \\
0 & C_{2} & D_{1} & 0 \\
c_{3} & 0 & 0 & d_{4}
\end{array}\right) \in S L(n+3, \mathbb{R}) \mid \cdots\right\} \simeq S(O(1,1) \times O(1, n)),
$$

and $\left(G_{1}, H_{1}, \eta\right)$ is of Cayley type. It is straightforward to check that $Z_{G_{1}}\left(X^{0}\right)$ consists of those elements in $H_{1}$ for which $a_{1} d_{4}-b_{2} c_{3}=1$, whereas for an arbitrary element $a_{1} d_{4}-b_{2} c_{3} \in\{ \pm 1\}$, i.e. $H_{1} / Z_{G_{1}}\left(X^{0}\right) \simeq \mathbb{Z} / 2 \mathbb{Z}$.

We choose $\sigma$ to be the inner automorphism

$$
\sigma=\operatorname{Ad}\left(\begin{array}{ccc}
1_{2} & & \\
& -1_{n} & \\
& & 1
\end{array}\right)
$$

then its fixed point group is

$$
G_{1}^{\sigma}=\left\{\left(\begin{array}{ccc}
A & B^{\prime} & 0 \\
C^{\prime} & D_{1}^{\prime} & 0 \\
0 & 0 & d_{4}
\end{array}\right) \in S L(n+3, \mathbb{R}) \mid \cdots\right\} \simeq S(O(2, n) \times O(1)),
$$


and one verifies condition (ii) of Theorem 5.1 for this involution. We have

$$
\begin{gathered}
G=\left\{\left(\begin{array}{ccc}
A & B^{\prime} & 0 \\
C^{\prime} & D_{1}^{\prime} & 0 \\
0 & 0 & 1
\end{array}\right) \mid\left(\begin{array}{cc}
A & B^{\prime} \\
C^{\prime} & D_{1}^{\prime}
\end{array}\right) \in S O_{0}(2, n)\right\}, \\
H=\left\{\left(\begin{array}{cccc}
a_{1} & 0 & 0 & 0 \\
0 & a_{4} & B_{3} & 0 \\
0 & C_{2} & D_{1} & 0 \\
0 & 0 & 0 & 1
\end{array}\right) \in G \mid \begin{array}{l}
a_{1} a_{4} \geq 1 \\
\operatorname{det} D_{1} \geq 1
\end{array}\right\},
\end{gathered}
$$

and $Z_{G}\left(X^{0}\right)=H \cap Z_{G_{1}}\left(X^{0}\right)$ is the subgroup of matrices with $a_{1}=1$, i.e. $Z_{G}\left(X^{0}\right) \simeq$ $S O_{0}(1, n)$ in the obvious manner. Therefore $\left(S O_{0}(2, n), S O_{0}(1, n), \tau\right)$ is a compactly causal subspace of $\left(G_{1}, Z_{G_{1}}\left(X^{0}\right), \eta\right)$ with a causal compactification $\Phi$ : $S O_{0}(2, n) / S O_{0}(1, n) \rightarrow S O(2, n+1) / P^{\prime}$ as in Theorem [5.1. Another treatment of this example can be found in [Mo 97].

For every Cayley space $\left(G_{1}, H_{1}, \eta\right)$ as in the theorem, there is a whole family of involutions $\sigma$ for which condition (i) can be easily verified.

Corollary 5.3. Let $\left(G_{1}, H_{1}, \eta\right)$ be an irreducible causal symmetric space of Cayley type. Then, for $\epsilon_{j} \in\{ \pm 1\}$, the inner involution $\sigma=\operatorname{Ad}\left(\exp \frac{\pi}{2} i \sum \epsilon_{j} H_{j}\right)$ commutes with $\eta$ and $\theta,(G, H, \tau)$ is a weak compactly causal subspace of $\left(G_{1}, H_{1}, \eta\right)$, and $\Phi: G / H \rightarrow G_{1} / P^{\prime}$ is a causal compactification.

Proof. An $\mathfrak{s l}(2)$-calculation shows $\sigma^{2}=\operatorname{Ad}\left(\exp \left(\pi i \sum \epsilon_{j} H_{j}\right)\right)=$ id. Since $\theta\left(i H_{j}\right)=$ $-i H_{j}$, and by another $\mathfrak{s l}(2)$-calculation $\eta\left(H_{j}\right)=-H_{j}$, the three involutions $\eta, \theta$ and $\sigma$ commute pairwise. Finally, from equation (10) - where we can replace $H_{j}$ also by $\epsilon_{j} H_{j}$ - it is immediate that $X_{j}, Y_{j} \in \mathfrak{q}_{1 \sigma}$.

Lemma 5.4. Let $(\mathfrak{g}, \mathfrak{k}, \theta)$ be irreducible Hermitian of noncompact type, $\rho$ an involution commuting with $\theta$. Then $\operatorname{dim} \mathfrak{z}\left(\mathfrak{g}^{\rho}\right) \leq 1$.

Proof. The Riemannian dual $\mathfrak{g}^{r}$ of $\left(\mathfrak{g}, \mathfrak{g}^{\rho}, \rho\right)$ is simple with $\mathfrak{g}_{\mathbb{C}}^{r}=\mathfrak{g}_{\mathbb{C}}$ simple, too. Therefore $\mathfrak{g}^{r}$ is irreducible orthogonal symmetric of type III ([He 78, p. 379]) and its maximal compact subalgebra $\mathfrak{g}^{\rho} \cap \mathfrak{k} \oplus i\left(\mathfrak{g}^{\rho} \cap \mathfrak{p}\right)$ has a center $\mathfrak{c}$ of dimension at most one. $\theta$ is an involution of this subalgebra, which implies $\mathfrak{c}=\mathfrak{g}^{\rho} \cap \mathfrak{k} \cap \mathfrak{c} \oplus i\left(\mathfrak{g}^{\rho} \cap \mathfrak{p}\right) \cap \mathfrak{c}$ and $\mathfrak{z}\left(\mathfrak{g}^{\rho}\right)=\mathfrak{g}^{\rho} \cap \mathfrak{k} \cap \mathfrak{c}+i\left(i\left(\mathfrak{g}^{\rho} \cap \mathfrak{p}\right) \cap \mathfrak{c}\right)$.

As $\sigma$ in the corollary is given in a very explicit form, it is possible to derive some additional information on the structure of $(\mathfrak{g}, \mathfrak{h}, \tau)$ for this case.

Proposition 5.5. Let $\left(\mathfrak{g}_{1}, \mathfrak{h}_{1}, \tau\right)$ be as in Corollary 5.3. Then:

(1) $\mathfrak{z}(\mathfrak{g})=\mathbb{R}\left(\sum \epsilon_{j} i H_{j}\right) \subset \mathfrak{q}_{1} \cap \mathfrak{k}_{1}$ and $\mathfrak{z}\left(\mathfrak{h}_{1}\right)=\mathbb{R} X^{0} \subset \mathfrak{p}_{1} \cap \mathfrak{q}_{1 \sigma}$.

(2) $(\mathfrak{g}, \theta, \tau) \simeq\left(\mathfrak{k}_{1}^{c}, \sigma, \eta\right)$, where $\mathfrak{k}_{1}^{c}:=\mathfrak{k}_{1} \cap \mathfrak{g} \oplus i\left(\mathfrak{k}_{1} \cap \mathfrak{q}_{1 \sigma}\right)$ is the $c$-dual of $\mathfrak{k}_{1}=$ $\mathfrak{k}_{1} \cap \mathfrak{g} \oplus \mathfrak{k}_{1} \cap \mathfrak{q}_{1 \sigma}$.

(3) $([\mathfrak{g}, \mathfrak{g}], \mathfrak{h}, \tau)$ is an irreducible symmetric space.

Proof. We have $\eta\left(H_{j}\right)=-H_{j}$ by an $\mathfrak{s l}(2)$-calculation, i.e. $H_{j} \in \mathfrak{q}_{1}$. The first statement then follows by applying Lemma 5.4 to the involutions $\sigma$ and $\eta$.

For the second claim we first prove that $(\mathfrak{g}, \theta) \simeq\left(\mathfrak{k}_{1}^{c}, \sigma\right)$. Defining

$$
A:=\frac{\pi}{2} i\left(\sum_{\left\{j \mid \epsilon_{j}=1\right\}} H_{j}-\sum_{\left\{j \mid \epsilon_{j}=-1\right\}} Y_{j}\right)
$$


by $\mathfrak{s l}(2)$-calculations we get $\operatorname{Ad}(\exp A)\left(H_{j}\right)=\epsilon_{j} H_{j}$ and $\operatorname{Ad}(\exp A)^{2}=$ id. Since $\theta=\operatorname{Ad}\left(\exp \frac{\pi}{2} i \sum H_{j}\right)$, we see from the definition of $\sigma$ that $\operatorname{Ad}(\exp A) \circ \theta=\sigma \circ$ $\operatorname{Ad}(\exp A)$. Therefore $\operatorname{Ad}(\exp A): \mathfrak{g}_{\mathbb{C}}=\left(\mathfrak{g}_{1}^{\sigma}\right)_{\mathbb{C}} \rightarrow \mathfrak{k}_{1 \mathbb{C}}$ is an isomorphism. Further, with $A \in \mathfrak{k}_{1}+i \mathfrak{p}_{1}$, the subalgebra $\mathfrak{k}_{1}+i \mathfrak{p}_{1}$ is trivially $\operatorname{Ad}(\exp A)$-invariant, and by this $\mathfrak{k}+i \mathfrak{p}=\mathfrak{g}_{\mathbb{C}} \cap\left(\mathfrak{k}_{1}+i \mathfrak{p}_{1}\right)$ is mapped onto $\mathfrak{k}_{1 \mathbb{C}} \cap\left(\mathfrak{k}_{1}+i \mathfrak{p}_{1}\right)=\mathfrak{k}_{1}$. Ad $(\exp A)$ also maps the $(-1)$-eigenspace of $\theta$ onto the (-1)-eigenspace of $\sigma$, i.e. $\operatorname{Ad}(\exp A)\left(i \mathfrak{p}_{1}\right)=\mathfrak{k}_{1} \cap \mathfrak{q}_{1 \sigma}$. As the map is complex linear, we finally get an isomorphism $\operatorname{Ad}(\exp A): \mathfrak{g}=$ $\mathfrak{k}+\mathfrak{p} \rightarrow \mathfrak{k}+i\left(\mathfrak{k}_{1} \cap \mathfrak{q}_{1 \sigma}\right)=\mathfrak{k}_{1}^{c}$.

To complete the proof we have to show that $\eta \circ \operatorname{Ad}(\exp A)=\operatorname{Ad}(\exp A) \circ \eta$. But $H_{j} \in \mathfrak{q}_{1}$; therefore $Y_{j}=\frac{1}{2}\left[i H_{j}, X_{j}\right] \in \mathfrak{q}_{1}$ and $\eta(A)=-A$, which is enough, as $\operatorname{Ad}(\exp A)$ is involutive.

For the last statement, with $\mathfrak{g}_{\mathbb{C}} \simeq\left(\mathfrak{k}_{1}^{c}\right)_{\mathbb{C}}=\mathfrak{k}_{1 \mathbb{C}}$, the classification of the Hermitian symmetric algebras $\left(\mathfrak{g}_{1}, \mathfrak{k}_{1}, \theta\right)([$ He 78, p. 518]) shows that $\mathfrak{g}=\mathfrak{z}(\mathfrak{g})+[\mathfrak{g}, \mathfrak{g}]$, with $[\mathfrak{g}, \mathfrak{g}]$ simple (and $\mathfrak{z}(\mathfrak{g})$ one dimensional) except for $\mathfrak{g}_{1}=\mathfrak{s u}(n, n)$. For this Lie algebra in its standard realization we can choose

$$
\eta\left(\left(\begin{array}{ll}
A & B \\
C & D
\end{array}\right)\right)=\left(\begin{array}{ll}
D & C \\
B & A
\end{array}\right),
$$

cf. section 7.1, and from this it is also clear that $([\mathfrak{g}, \mathfrak{g}], \mathfrak{h}, \tau) \simeq(\tilde{\mathfrak{g}} \oplus \tilde{\mathfrak{g}}, \Delta(\tilde{\mathfrak{g}} \oplus \tilde{\mathfrak{g}}), \tilde{\tau})$ with $\tilde{\mathfrak{g}}$ simple and $\tilde{\tau}: \tilde{\mathfrak{g}} \oplus \tilde{\mathfrak{g}} \rightarrow \tilde{\mathfrak{g}} \oplus \tilde{\mathfrak{g}},(X, Y) \mapsto(Y, X)$.

Example 5.6. Choosing all $\epsilon_{i}=+1$ (or -1 ), we get $\sigma=\theta$, and our "compactification" is the causal diffeomorphism $K_{1} / K_{1} \cap H_{1} \rightarrow G_{1} / P^{\prime}$.

Example 5.7 (Causal compactification of $U(p, q) / O(p, q)$ ). With

$$
G_{1}=\left\{\begin{array}{ll}
\left(\frac{A}{B} \frac{B}{A}\right) \in S U(n, n) & \begin{array}{l}
A, B \in M(n \times n, \mathbb{C}), \\
{ }^{t} A-{ }^{t} B \bar{B}=1,{ }^{t} A \bar{B}={ }^{t} B A
\end{array}
\end{array}\right\} \simeq S p(n, \mathbb{R}),
$$

the Cartan involution $\theta(g)={ }^{t} \bar{g}^{-1}$, and the Cartan algebra $\mathfrak{t}$ given by the set of diagonal matrices in $\mathfrak{g}_{1}$, we choose $E_{j}=E_{j, j+n}$ and $E_{-j}=E_{j+n, j}$ for $j=$ $1, \ldots, n$, where $E_{k, l}$ is the matrix with entry 1 in the $k$-th row and $l$-th column and otherwise zero. Then $\eta=\operatorname{Ad}\left(\exp \frac{\pi}{2} i X^{0}\right)$ is given by complex conjugation, and for the subgroup of real matrices $H_{1}$ we have an isomorphism

$$
H_{1} \rightarrow G L(n, \mathbb{R}),\left(\begin{array}{cc}
A & B \\
B & A
\end{array}\right) \mapsto A+B .
$$

Choosing $\epsilon_{j}=+1$ for $j=1, \ldots, p$, and $\epsilon_{j}=-1$ for the remaining $j$, for the fixed point group of $\sigma$ we get

$$
G=\left\{\left(\begin{array}{cccc}
A_{1} & 0 & 0 & B_{2} \\
0 & A_{4} & B_{3} & 0 \\
0 & \bar{B}_{2} & \bar{A}_{1} & 0 \\
\bar{B}_{3} & 0 & 0 & \bar{A}_{4}
\end{array}\right) \mid \begin{array}{l}
A_{1} \in M(p \times p, \mathbb{C}), A_{4} \in M(q \times q, \mathbb{C}), \\
B_{2} \in M(p \times q, \mathbb{C}), B_{3} \in M(q \times p, \mathbb{C}), \\
{ }^{t} A-{ }^{t} B \bar{B}=I,{ }^{t} A \bar{B}={ }^{t} \bar{B} A
\end{array}\right\},
$$

where $p+q=n$. It is straightforward to check that

$$
\rho: G \rightarrow U(p, q),\left(\begin{array}{cc}
A & \frac{B}{A}
\end{array}\right) \mapsto\left(\begin{array}{ll}
A_{1} & B_{2} \\
B_{3} & \bar{A}_{4}
\end{array}\right),
$$

is a group isomorphism, and that the subgroup of real matrices $H$ is mapped onto $O(p, q)$.

We give the causal compactification in the Harish-Chandra realization of the Bergman-Šilov boundary. Remember that - with the usual choice of positive 
roots compatible with our choice of the $E_{ \pm j}$ 's - the decomposition in $P_{1}^{+}, K_{1 \mathbb{C}}$ and $P_{1}^{-}$-components for $G_{1}$ is given by

$$
\left(\begin{array}{ll}
\frac{A}{B} & \bar{A}
\end{array}\right)=\left(\begin{array}{cc}
I & B \bar{A}^{-1} \\
0 & I
\end{array}\right)\left(\begin{array}{cc}
A-B \bar{A}^{-1} \bar{B} & 0 \\
0 & \bar{A}
\end{array}\right)\left(\begin{array}{cc}
I & 0 \\
\bar{A}^{-1} B & I
\end{array}\right),
$$

and the operation of $G_{1}$ on $P_{1}^{+}$by

$$
\left(\begin{array}{ll}
A & B \\
\bar{B} & \bar{A}
\end{array}\right)\left(\begin{array}{cc}
I & Z \\
0 & I
\end{array}\right) K_{1 \mathbb{C}} P_{1}^{-}=\left(\begin{array}{cc}
I & (A Z+B)(\bar{B} Z+\bar{A})^{-1} \\
0 & I
\end{array}\right) K_{1 \mathbb{C}} P_{1}^{-} .
$$

By these equations, and inverting $\rho$, we now easily get the causal compactification

$$
\begin{aligned}
U(p, q) / O(p, q) & \rightarrow \check{S}_{1} \\
\left(\begin{array}{ll}
A_{1} & B_{2} \\
\bar{B}_{3} & \bar{A}_{4}
\end{array}\right) O(p, q) & \mapsto\left(\begin{array}{cc}
-A_{1} & B_{2} \\
B_{3} & -A_{4}
\end{array}\right)\left(\begin{array}{cc}
\bar{A}_{1} & -\bar{B}_{2} \\
-\bar{B}_{3} & \bar{A}_{4}
\end{array}\right)^{-1},
\end{aligned}
$$

where the Bergman-ŠSilov boundary is given by $\breve{S}_{1}=\{Z \in M(n \times n, \mathbb{C}) \mid \bar{Z} Z=$ $I,{ }^{t} Z=Z$ \} (Wo 72, He 78, p. 527]).

In fact, in proving Theorem 5.1 we used only the compactly causal structure of the Cayley space $G_{1} / H_{1}$. In some cases it is therefore possible to construct causal compactifications while assuming $G_{1} / H_{1}$ to be only compactly causal.

Definition 5.8. Let $\left(\mathfrak{g}_{1}, \mathfrak{k}_{1}, \theta\right)$ be an irreducible orthogonal Lie algebra of noncompact Hermitian type. Let $\left\{\gamma_{1}, \ldots, \gamma_{r}\right\}$ be a maximal system of strongly orthogonal noncompact roots, and $E_{ \pm j} \in \mathfrak{g}_{1 \mathbb{C}, \pm \gamma_{j}}, j=1, \ldots, r$, vectors with $E_{j}+$ $E_{-j}, i E_{j}-i E_{-j} \in \mathfrak{g}_{1}$. Let $\tau$ be an involutive automorphism of $\mathfrak{g}_{1}$ commuting with $\theta$. The set $\left\{E_{ \pm j} \mid 1 \leq j \leq r\right\}$ is called a system of first kind with respect to $\tau$ if $\tau\left(E_{ \pm j}\right)=-E_{\mp j}$ for all $j$. The set is called a system of second kind if $r=2 r^{\prime}$, and $\tau\left(E_{ \pm j}\right)=-E_{\mp\left(j+r^{\prime}\right)}$ or $\tau\left(E_{ \pm\left(j+r^{\prime}\right)}\right)=-E_{\mp j}$, for $j=1, \ldots, r^{\prime}$.

Theorem 5.9. Let $\left(G_{1}, H_{1}, \tau\right), H_{1}:=G_{1}^{\tau}$, be an irreducible compactly causal symmetric space with $G_{1} \subset G_{1 \mathbb{C}}, G_{1 \mathbb{C}}$ the simply connected Lie group corresponding to $\mathfrak{g}_{1 \mathbb{C}}$, and $\left(G_{1}, K_{1}, \theta\right)$ Hermitian symmetric of tube type. Define $\sigma:=\eta \circ \tau$, and assume that one of the following cases holds:

(i) The $\left\{E_{ \pm j} \mid 1 \leq j \leq r\right\}$ form a system of first kind with respect to $\tau$.

(ii) The $\left\{E_{ \pm j} \mid 1 \leq j \leq r\right\}$ form a system of second kind and $\left(\sum \mathbb{R} X_{j}\right) \cap \mathfrak{q}_{1 \sigma}$ is maximal abelian in $\mathfrak{p}_{1} \cap \mathfrak{q}_{1 \sigma}$.

Then $(G, H, \tau):=\left(\left(G_{1}^{\sigma}\right)_{0}, H_{1} \cap\left(G_{1}^{\sigma}\right)_{0}, \tau\right)$ is a weak compactly causal subspace of $\left(G_{1}, G_{1}^{\eta}, \eta\right)$, and $\Phi: G / H \rightarrow G_{1} / P^{\prime}$ is a causal compactification of $i t$.

Proof. With $\tau\left(X^{0}\right)=\theta\left(X^{0}\right)=-X^{0}$ we see immediately that $\sigma^{2}=$ id and that this involution commutes with $\tau$ and $\theta$. From the Langlands decomposition we have $P^{\prime} \simeq Z_{G_{1}}\left(X^{0}\right) \times Q_{1}^{-}$, and $\sigma\left(Q_{1}^{-}\right)=Q_{1}^{+}$by $\sigma\left(X^{0}\right)=\tau\left(X^{0}\right)=-X^{0}$. On the other hand, by this the centralizer $Z_{G_{1}}\left(X^{0}\right)$ is $\sigma$ - and $\tau$-invariant, and therefore $P^{\prime} \cap G=Z_{G_{1}}\left(X^{0}\right) \cap G$. In fact, our assumptions assure that $\left(G_{1}, G_{1}^{\eta}, \eta\right)$ is of Cayley type with $G_{1}^{\eta}=Z_{G_{1}}\left(X^{0}\right)$. As $\sigma=\eta \tau$ and $H_{1}=G_{1}^{\tau}$, we have $H \subset G_{1}^{\eta}$, and that $P^{\prime} \cap G=H$. Now, by the same arguments as in the proof of Theorem 5.1 the map $\Phi$ is an injective submersion.

We show next the density of the image, again by using Matsuki's double coset decomposition. For systems of the first kind we have $\mathfrak{a}=\sum \mathbb{R} X_{j} \subset \mathfrak{p}_{1} \cap \mathfrak{q}_{1 \sigma}$, 
$W_{\sigma}(\mathfrak{a})=W(\mathfrak{a})$, and the open double cosets are $G w_{v} P\left(\mathfrak{a}, \Sigma^{+}\right)$, where $w_{v}$ represents the element $v \in W\left(\mathfrak{a}, K_{1} \cap G\right) \backslash W(\mathfrak{a})$.

In contrast, for systems of the second kind, by condition (i) of Proposition 4.1 the set $\mathfrak{a} \cap \mathfrak{q}_{1 \sigma}=\sum_{j=1}^{r^{\prime}} \mathbb{R}\left(X_{j}+X_{j+r^{\prime}}\right)$ must be maximal abelian in $\mathfrak{p}_{1} \cap \mathfrak{q}_{1 \sigma}$. With $\mathfrak{a} \cap \mathfrak{g}=\sum \mathbb{R}\left(X_{j}-X_{j+r^{\prime}}\right)$ and $W(\mathfrak{a}) \simeq(\mathbb{Z} / 2 \mathbb{Z})^{r} \times S_{r}$, cf. Corollary 2.2, we see that $W_{\sigma}(\mathfrak{a})$ is generated by all permutations of the pairs $\left(X_{1}, X_{1+r^{\prime}}\right), \ldots,\left(X_{r^{\prime}}, X_{r^{\prime}+r^{\prime}}\right)$, the transpositions $\left\langle X_{j}, X_{j+r^{\prime}}\right\rangle$ - which together form a semidirect product of permutations $S_{r^{\prime}} \times(\mathbb{Z} / 2 \mathbb{Z})^{r^{\prime}}$ — and the reflections which map $X_{j}+X_{j+r^{\prime}}$ onto its negative and leave all vectors orthogonal to this one fixed. According to Theorem 2.1 and the following discussion, we have $\Sigma(\mathfrak{a}) \backslash \Sigma(\mathfrak{a} \cap \mathfrak{g})=\left\{ \pm \frac{1}{2}\left(\gamma_{s}^{c}+\gamma_{t}^{c}\right), \pm \frac{1}{2}\left(\gamma_{u}^{c}-\gamma_{v}^{c}\right) \mid\right.$ $\left.1 \leq s, t, u, v \leq r, u \neq v,|u-v| \neq r^{\prime}\right\}$ and a $\sigma \theta$-compatible positive subset in the sense of Proposition 4.1 can be defined using the lexicographic ordering $\gamma_{1}^{c}>\gamma_{1+r^{\prime}}^{c}>\gamma_{2}^{c}>\gamma_{2+r^{\prime}}^{c}>\ldots>\gamma_{r^{\prime}+r^{\prime}}^{c}$. Of course, also in this case all other $\sigma \theta$-compatible positive systems are $W_{\sigma}(\mathfrak{a})$-conjugate to the given one.

We determine the open double cosets in $G P^{\prime}$ by Theorem 4.2. As in the proof of Theorem [5.1, a minimal parabolic subalgebra $\mathfrak{p}\left(\mathfrak{a}, w \Sigma(\mathfrak{a})^{+}\right)$, where $\left\langle\Sigma(\mathfrak{a})^{+}, \mathfrak{a}_{+}^{\prime}\right\rangle \subset$ $\mathbb{R}^{+}$, lies in $\mathfrak{p}^{\prime}$ if and only if $w \in W(\mathfrak{a})_{\mathfrak{m}^{\prime}}$. Hence the classes of subalgebras in open orbits are parametrized by $W(\mathfrak{a})_{\mathfrak{m}^{\prime}} \cap W_{\sigma}(\mathfrak{a})$. If we restrict our attention to open double cosets and divide out algebras within the same orbit, we get the diagram

$$
\begin{aligned}
W(\mathfrak{a})_{\mathfrak{m}^{\prime}} & \cap W_{\sigma}(\mathfrak{a}) \rightarrow W_{\sigma}(\mathfrak{a}) \rightarrow W\left(\mathfrak{a}, K_{1} \cap G\right) \backslash W_{\sigma}(\mathfrak{a}) \\
& \downarrow \\
W(\mathfrak{a})_{\mathfrak{m}^{\prime}} & \cap W\left(\mathfrak{a}, K_{1} \cap G\right) \backslash W(\mathfrak{a})_{\mathfrak{m}^{\prime}} \cap W_{\sigma}(\mathfrak{a})
\end{aligned}
$$

where we have to prove that $\varphi$ is surjective. For spaces of first kind $Y_{j} \in \mathfrak{p}_{1} \cap \mathfrak{q}_{1 \sigma}$, and the arguments given in the proof of Theorem 5.1 apply without any change. In the other case we have to show that the reflections with respect to $X_{j}+X_{j+r^{\prime}}$ are elements of $W\left(\mathfrak{a}, K_{1} \cap G\right)$. But now $X_{j}+X_{j+r^{\prime}}$ and $Y_{j}+Y_{j+r^{\prime}}$ are in $\mathfrak{p}_{1} \cap \mathfrak{q}_{1 \sigma}$; therefore

$$
\left[X_{j}+X_{j+r^{\prime}}, Y_{j}+Y_{j+r^{\prime}}\right]=-2 i\left(H_{j}+H_{j+r^{\prime}}\right) \in \mathfrak{k}_{1} \cap \mathfrak{g}
$$

and $\operatorname{Ad}\left(\exp \frac{\pi}{2} i\left(H_{j}+H_{j+r^{\prime}}\right)\right)$ is a representative of the reflection we seek.

It remains to prove the causality of $G / H$ and $\Phi$. First of all we see that $\tau\left(Y_{j}\right)=$ $Y_{j}$, and for systems of the second kind $\tau\left(Y_{j}\right)=Y_{j+r^{\prime}}$ and $\tau\left(Y_{j+r^{\prime}}\right)=Y_{j}$. In both cases we have $\tau\left(Y^{0}\right)=Y^{0}$. Thus $Z^{0}=\frac{1}{4}\left[X^{0}, Y^{0}\right] \in \mathfrak{q}_{1}$, and this shows that $\tau\left(Y_{ \pm}\right)=\tau\left(\frac{1}{2} Y^{0} \mp Z^{0}\right)=Y_{\mp}$. With $\operatorname{Ad}\left(\exp \frac{\pi}{2} i X^{0}\right)\left(Y^{0}\right)=-Y^{0}$, we then conclude that $\sigma\left(Y_{ \pm}\right)=-Y_{\mp}$, showing the $\sigma$-invariance of the cone $C_{k}$. For the Cayley space $\left(G_{1}, G_{1}^{\eta}, \eta\right)$ the compactly causal structure is defined by $C_{k} \subset \mathfrak{q}_{1}$. By $\sigma=\eta \circ \tau$ we have $\left.\tau\right|_{\mathfrak{g}}=\left.\eta\right|_{\mathfrak{g}}$ and $\tau|G=\eta| G$, and, applying Lemma 3.1, the cone $\operatorname{pr}_{\mathfrak{g}} C_{k} \subset \mathfrak{q}_{1} \cap \mathfrak{g}$ makes $G / H$ into a weak compactly causal subspace of $G_{1} / G_{1}^{\eta}$. This also ensures that $\Phi$ is a causal map.

Example 5.10. For $\left(G_{1}, H_{1}, \tau\right)$ of Cayley type, $\tau\left(E_{ \pm j}\right)=E_{ \pm j}$. Defining $\tilde{E}_{+j}:=$ $i E_{+j}$ and $\tilde{E}_{-j}:=-i E_{-j}$ gives a system of the first kind. Applying Theorem 5.9 we recover the "compactification" $\Phi: K_{1} / H_{1} \cap K_{1} \rightarrow \check{S}_{1}$.

Example 5.11 (Causal compactification of spaces of Cayley type). Let $\left(\mathfrak{g}_{1}, \mathfrak{h}_{1}, \tau\right)$ be compact causal of group type, i.e. $\left(\mathfrak{g}_{1}, \mathfrak{h}_{1}, \tau\right)=(\tilde{\mathfrak{g}} \times \tilde{\mathfrak{g}}, \Delta(\tilde{\mathfrak{g}} \times \tilde{\mathfrak{g}}), \tau)$, with involution $\tau: \tilde{\mathfrak{g}} \times \tilde{\mathfrak{g}} \rightarrow \tilde{\mathfrak{g}} \times \tilde{\mathfrak{g}}, \quad(X, Y) \mapsto(Y, X)$, and $\Delta(\tilde{\mathfrak{g}} \times \tilde{\mathfrak{g}}):=\{(X, X) \mid X \in \tilde{\mathfrak{g}}\}$. Then $\left(G_{1}, K_{1}, \theta_{1}\right), \theta_{1}:=\theta \times \theta$, is Hermitian symmetric of tube type if and only if $(\tilde{G}, \tilde{K}, \theta)$ is. If we choose the $\left\{\tilde{E}_{ \pm j} \mid j=1, \ldots, r^{\prime}\right\}$ in $\tilde{\mathfrak{g}}_{\mathbb{C}}$ with the usual properties, then 
$E_{ \pm j}:=\left(\tilde{E}_{ \pm j}, 0\right)$ and $E_{ \pm\left(j+r^{\prime}\right)}:=\left(0,-\tilde{E}_{\mp j}\right)$ form a system of the second kind. Theorem 5.9 then gives $G=\{(g, \tilde{\eta}(g)) \mid g \in \tilde{G}\}$, where $\tilde{\eta}:=\operatorname{Ad}\left(\exp \frac{\pi}{2} i \tilde{X}^{0}\right)$, and therefore $(G, H, \tau) \simeq\left(\tilde{G}, \tilde{G}^{\tilde{\eta}}, \tilde{\eta}\right)$, which is of Cayley type. This gives us a variant of the causal compactification for Cayley spaces already constructed by different methods, cf. [ÓØ 97 Ko 93 Ka 87.

Example 5.12 (Causal compactification of $S O^{*}(2 n) / O(n, \mathbb{C})$ ). For

$$
G_{1}=\left\{\left(\begin{array}{ll}
A & B \\
C & D
\end{array}\right) \in S L(2 n, \mathbb{C}) \mid \begin{array}{l}
{ }^{t} A \bar{A}-{ }^{t} C \bar{C}=I,{ }^{t} A \bar{B}={ }^{t} C \bar{D} \\
{ }^{t} D \bar{D}-{ }^{t} B \bar{B}=I
\end{array}\right\}=S U(n, n),
$$

let $\tau$ be complex conjugation and correspondingly $H_{1} \simeq O(n, n)$, the subgroup of real matrices. (Note that $\left(G_{1}, H_{1}, \tau\right)$, as the $\rho$-image of the semisimple symmetric space $([G, G], H, \tau)$ defined in Example [5.7 is compactly causal.) Then $E_{+j}=$ $i E_{j, j+n}$ and $E_{-j}=-i E_{j+n, j}$ form a system of first kind. The involution $\sigma$ has as fixed point group

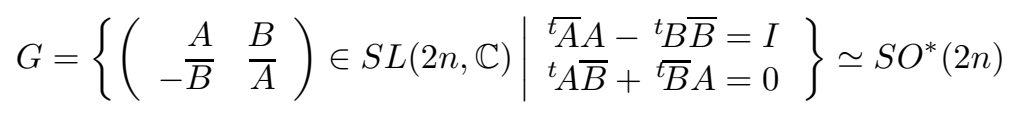

([He 78, p. 527]), and $H$ is the subgroup of all real matrices with

$$
H \rightarrow O(n, \mathbb{C}),\left(\begin{array}{rr}
A & B \\
-B & A
\end{array}\right) \mapsto A+i B,
$$

an isomorphism. In the Harish-Chandra realization we now get

$$
\begin{aligned}
& S O^{*}(2 n) / O(n, \mathbb{C}) \rightarrow \check{S}_{1} \\
& \left(\begin{array}{rr}
A & B \\
-\bar{B} & \bar{A}
\end{array}\right) H \mapsto(-i A+B)(i \bar{B}+\bar{A})^{-1},
\end{aligned}
$$

where $\check{S}_{1}=\left\{Z \in M(n \times n, \mathbb{C}) \mid{ }^{\natural} Z Z=I\right\}$ is the Bergman-ŠSilov boundary of $S U(n, n) / S(U(n) \times U(n))$.

\section{Compactly Causal spaces with no compactification}

Using our results of the last section, it is easy to construct causal compactifications for the spaces listed in the introduction. For those with "no compactification" causal compactifications can exist for some small $n$ from the existence of special isomorphisms, cf. [He 78, p. 519f]. We will prove here the nonexistence of further compactifications as symmetric orbits by making use of the classification of open symmetric orbits in symmetric $R$-spaces ([Ma 73]).

For this we note first that the Bergman-Šilov boundary $\check{S}_{1}$ of a Hermitian symmetric space of tube type is such a space, since, by Theorem [2.3] it is an $R$-space, i.e., a quotient $G_{1} / P^{\prime}$ of a semisimple symmetric group by a parabolic subgroup, and in addition $\left(K_{1}, K_{1} \cap P^{\prime}, \eta\right)$ and $\check{S}_{1}=K_{1} / K_{1} \cap P^{\prime}$ possess $K_{1}$-invariant Riemannian structures ([KW 65, Thm. 4.9]). An open orbit of $G \subset G_{1}$ is called symmetric if it is a symmetric subspace of $G_{1} / P^{\prime}$. We remark that our causal compactifications already constructed are symmetric orbits.

Proposition 6.1. For the following compactly causal symmetric Lie algebras $(\mathfrak{g}, \mathfrak{h})$ there exists no compactly causal space $\left(G_{1}, H_{1}, \tau\right)$ such that the subspace $(G, H, \tau)$ associated to $(\mathfrak{g}, \mathfrak{h}) \subset\left(\mathfrak{g}_{1}, \mathfrak{h}_{1}\right)$ is a compactly causal subspace and $\Phi: G / H \rightarrow G_{1} / P^{\prime}$, with $P^{\prime}$ appropriately chosen, a causal compactification as symmetric orbit. 
$\mathfrak{g}$

$$
\begin{aligned}
& \mathfrak{s o}(2, p+q) \\
& \mathfrak{e}_{6(-14)} \\
& \mathfrak{e}_{7(-25)} \\
& \mathfrak{s o}(2, n) \oplus \mathfrak{s o}(2, n) \\
& \mathfrak{e}_{6(-14)} \oplus \mathfrak{e}_{6(-14)} \\
& \mathfrak{e}_{7(-25)} \oplus \mathfrak{e}_{7(-25)}
\end{aligned}
$$

$\mathfrak{h}$

$$
\begin{array}{ll}
\mathfrak{s o}(p, 1) \oplus \mathfrak{s o}(1, q) & \min (p, q) \geq 2,(p, q) \neq(2,2),(3,3) \\
\mathfrak{s p}(2,2) & \\
\mathfrak{s u}^{*}(8) & n=5 \text { or } n \geq 7 \\
\mathfrak{s o}(2, n) & \\
\mathfrak{e}_{6(-14)} & \\
\mathfrak{e}_{7(-25)} &
\end{array}
$$

Proof. Assume to the contrary that $G / H \subset G_{1} / P^{\prime}$ is a symmetric orbit. When we decompose $G_{1} / P^{\prime}=\tilde{S}_{1} \times \ldots \times \tilde{S}_{n}$ into irreducible symmetric $R$-spaces, $G=$ $\tilde{G}_{1} \times \ldots \times \tilde{G}_{m}$ decomposes also into factors, where each factor gives a symmetric orbit in $\tilde{S}_{j}$ or $\tilde{S}_{j} \times \tilde{S}_{j+1}$, with $\tilde{S}_{j} \simeq \tilde{S}_{j+1}$ (Ma 73] $)$. By looking at the classification] of symmetric orbits $G / H \rightarrow \widetilde{S}_{1}$, we see that no space on our list - with the exception of the already mentioned low dimensional cases - possesses such a compactification. For the last three cases with $G=G_{0} \times G_{0}$, a compactification may be constructed from maps $G_{0} \times\{e\} \rightarrow \tilde{S}_{1}$ and $\{e\} \times G_{0} \rightarrow \tilde{S}_{2}$. However then $\mathfrak{h} \simeq \mathfrak{g}_{0}$ would not be simple, which is the case only for $\mathfrak{s o}(2,2) \simeq \mathfrak{s l}(2, \mathbb{R}) \oplus \mathfrak{s l}(2, \mathbb{R})$.

It remains to check the case $G \rightarrow \breve{S}_{1} \simeq \tilde{S} \times \tilde{S}$. For spaces of tube type the dimension of the Bergman-Šilov boundary $\check{S}_{1}$ is $\frac{1}{2} \operatorname{dim}\left(G_{1} / K_{1}\right)$, and we get the necessary condition $\operatorname{dim} \mathfrak{g}-\operatorname{dim} \mathfrak{h}=\frac{1}{2}\left(\operatorname{dim} \mathfrak{g}_{1}-\operatorname{dim} \mathfrak{k}_{1}\right)$. Moreover, for $G$ simple the only possible imbedding is the diagonal imbedding $G \rightarrow \Delta(G) \subset G \times G$ or a modification of this map as in Example 5.11 ( Ma 73]). For the first three groups of our list we therefore have the condition $\operatorname{dim} \mathfrak{h}=\operatorname{dim} \mathfrak{k}$, and this equation can only be solved for $\mathfrak{s o}(2, p+q)$ with $p=1$ or $q=1$, recovering the compactification for the spaces of Cayley type. For the last three groups a compactification would give us an imbedding $\mathfrak{g}_{0} \oplus \mathfrak{g}_{0} \hookrightarrow \mathfrak{g}_{1} \oplus \mathfrak{g}_{1}$, with $\left(\mathfrak{g}_{1}, \mathfrak{k}_{1}, \theta\right)$ Hermitian symmetric of tube type, such that $\Delta\left(\mathfrak{g}_{0} \oplus \mathfrak{g}_{0}\right)$ goes into the sum $\mathfrak{p}^{\prime} \oplus \tilde{\mathfrak{p}}^{\prime}$ of two maximal parabolic subalgebras. With the Langlands decomposition $\mathfrak{p}^{\prime}=\mathfrak{m}^{\prime}+\mathbb{R} X^{0}+\mathfrak{q}_{1}^{-}$we get from this an imbedding $\mathfrak{g}_{0} \hookrightarrow \mathfrak{m}^{\prime}(\overline{\text { Va } 84}$, Cor. 3.14.3]) with $\theta$-invariant image ([Wa 72. Lemma 1.1.5.5]). Therefore the rank and real rank of $\mathfrak{m}^{\prime}$ are restricted from below by the corresponding quantities of $\mathfrak{g}_{0}$, excluding $\mathfrak{g}_{1}=\mathfrak{s o}(2, k)$. Using our equation on the dimensions, we are left with $\mathfrak{g}_{0}=\mathfrak{s o}(2, n)$ and $\mathfrak{g}_{1}=\mathfrak{s p}(k, \mathbb{R})$ or $\mathfrak{g}_{1}=\mathfrak{s o}^{*}(4 k), k \geq$ 3 . For the first $\mathfrak{g}_{1}$, equality of dimensions leads to $(n+1)(n+2)=2 k(k+1)$, whereas from $\mathfrak{g}_{0} \hookrightarrow \mathfrak{m}^{\prime}=\mathfrak{s l}(k, \mathbb{R})$ necessarily $(n+1)(n+2) \leq 2\left(k^{2}-1\right)$, a contradiction. For the second $\mathfrak{g}_{1}$, comparing the dimensions gives $n \geq 2 k$; but $\mathfrak{g}_{0} \hookrightarrow \mathfrak{m}^{\prime}=\mathfrak{s u}^{*}(2 k)$, mapping maximal compact subalgebras into compact subalgebras, gives $1+\left[\frac{n}{2}\right]<k$, a contradiction, too.

\section{Causal compactifications for the Classical Causal spaces}

To be complete, we give here some details for the compactification of the classical symmetric spaces not already treated in the various examples. For the definition of these spaces as well as for our notation we follow [He 78, Chapter X].

\footnotetext{
${ }^{2}$ According to W. Bertram (personal communication), the classification in [Ma 73] is not complete. However, the part of the classification needed by us is not affected by this.
} 
7.1. The compactification of $S(U(p, q) \times U(q, p)) / S U(p, q)$. The group

$$
G_{1}=\left\{\left(\begin{array}{ll}
A & B \\
C & D
\end{array}\right) \in S L(2 n, \mathbb{C}) \mid \begin{array}{l}
{ }^{t} A \bar{A}-{ }^{t} C \bar{C}=I,{ }^{t} A \bar{B}={ }^{t} C \bar{D} \\
{ }^{t} D \bar{D}-{ }^{t} B \bar{B}=I
\end{array}\right\}=S U(n, n)
$$

verifies the conditions of Corollary [5.3. The real rank of $S U(n, n)$ is $n$, and we can use the same system of strongly orthogonal roots as for $S p(n, \mathbb{R})$ in Example 5.7 However, $\eta$ is no longer given by complex conjugation, but

$$
\eta\left(\left(\begin{array}{ll}
A & B \\
C & D
\end{array}\right)\right)=\left(\begin{array}{ll}
0 & I \\
I & 0
\end{array}\right)\left(\begin{array}{ll}
A & B \\
C & D
\end{array}\right)\left(\begin{array}{ll}
0 & I \\
I & 0
\end{array}\right)=\left(\begin{array}{ll}
D & C \\
B & A
\end{array}\right) .
$$

Then

$$
H_{1}=\left\{\begin{array}{l|l}
\left(\begin{array}{ll}
A & B \\
B & A
\end{array}\right) \in S L(2 n, \mathbb{C}) & \begin{array}{l}
{ }^{t} A \bar{A}-{ }^{t} B \bar{B}=I, \\
{ }^{t} A \bar{B}={ }^{t} B \bar{A}
\end{array}
\end{array}\right\} \simeq S L(n, \mathbb{C}) \times \mathbb{R}^{+} I,
$$

where the isomorphism is the same as that for $H_{1}$ in Example 5.7

Again let $\epsilon_{j}=+1$ for $j=1, \ldots, p$, and $\epsilon_{j}=-1$ for $j=p+1, \ldots, n$. Then for the fixed point group of $\sigma$ we get

$$
\begin{gathered}
G=\left\{\left(\begin{array}{cccc}
A_{1} & 0 & 0 & B_{2} \\
0 & A_{4} & B_{3} & 0 \\
0 & C_{2} & D_{1} & 0 \\
C_{3} & 0 & 0 & D_{4}
\end{array}\right) \in S U(n, n) \mid \begin{array}{l}
A_{1}, D_{1} \in M(p \times p, \mathbb{C}) \\
A_{4}, D_{4} \in M(q \times q, \mathbb{C}) \\
B_{2}, C_{2} \in M(q \times p, \mathbb{C}) \\
B_{3}, C_{3} \in M(p \times q, \mathbb{C})
\end{array}\right\} \\
\simeq\left\{\left(\left(\begin{array}{ll}
A_{1} & B_{2} \\
C_{3} & D_{4}
\end{array}\right),\left(\begin{array}{cc}
A_{4} & B_{3} \\
C_{2} & D_{1}
\end{array}\right)\right) \begin{array}{l}
{ }^{t} \bar{A}_{k} A_{k}-{ }^{t} C_{m} C_{m}=I,{ }^{t} \bar{D}_{n} D_{n}-{ }^{t} \bar{B}_{l} B_{l}=I \\
(k, l, m, n) \in\{(1,2,3,4),(4,3,2,1)\}
\end{array}\right. \\
=S(U(p, q) \times U(q, p)) .
\end{gathered}
$$

The subgroup $H=G \cap H_{1}$ maps under the same isomorphism onto

$$
\tilde{H}=\left\{\left(\left(\begin{array}{ll}
A_{1} & B_{2} \\
B_{3} & A_{4}
\end{array}\right),\left(\begin{array}{ll}
A_{4} & B_{3} \\
B_{2} & A_{1}
\end{array}\right)\right) \mid \ldots\right\} \simeq S U(p, q)
$$

and we get the causal compactification

$$
\begin{aligned}
& S(U(p, q) \times U(q, p)) / \tilde{H} \rightarrow \check{S}_{1} \\
&\left(\left(\begin{array}{ll}
A_{1} & B_{2} \\
C_{3} & D_{4}
\end{array}\right),\left(\begin{array}{ll}
A_{4} & B_{3} \\
C_{2} & D_{1}
\end{array}\right)\right) \tilde{H} \mapsto\left(\left(\begin{array}{cc}
-A_{1} & B_{2} \\
B_{3} & -A_{4}
\end{array}\right)\left(\begin{array}{cc}
D_{1} & -C_{2} \\
-C_{3} & D_{4}
\end{array}\right)^{-1}\right),
\end{aligned}
$$

where the Bergman-Šilov boundary of $S U(n, n) / S(U(n) \times U(n))$ in the HarishChandra realization is given by $\check{S}_{1}=\left\{Z \in M(n \times n, \mathbb{C}) \mid{ }^{t} Z \bar{Z}=I\right\}$ (Wo 72 He 78] $)$.

7.2. The compactification of $U(2 p, 2 q) / S p(p, q)$. We apply Corollary 5.3 to

$$
G_{1}=\left\{\left(\begin{array}{cc}
A & B \\
-\bar{B} & \bar{A}
\end{array}\right) \in S L(4 n, \mathbb{C}) \begin{array}{l}
{ }^{t} A \bar{A}-{ }^{t} \bar{B} B=I \\
{ }^{t} A \bar{B}+{ }^{t} \bar{B} A=0
\end{array}\right\} \simeq S O^{*}(4 n),
$$

with Cartan algebra $\mathfrak{t}=\left\{i \operatorname{diag}\left(x_{1}, \ldots, x_{2 n},-x_{1}, \ldots,-x_{2 n}\right) \mid x_{j} \in \mathbb{R}\right\}$, and

$$
E_{j}=\left(\begin{array}{cc}
0 & E_{j, j+n}-E_{j+n, j} \\
0 & 0
\end{array}\right) \text { and } \quad E_{-j}=\left(\begin{array}{cc}
0 & 0 \\
-E_{j, j+n}+E_{j+n, j} & 0
\end{array}\right),
$$

$j=1, \ldots, n$. With the given data we have

$$
\eta\left(\left(\begin{array}{cc}
A & B \\
-\bar{B} & \bar{A}
\end{array}\right)\right)=\left(\begin{array}{cc}
-J_{n} \bar{A} J_{n} & -J_{n} \bar{B} J_{n} \\
J_{n} B J_{n} & -J_{n} A J_{n}
\end{array}\right) \text {, where } J_{n}:=\left(\begin{array}{cc}
0 & I_{n} \\
-I_{n} & 0
\end{array}\right) .
$$


The fixed point group of $\eta$ is

$$
H_{1}=\left\{\left(\begin{array}{rrrr}
A_{1} & A_{2} & B_{1} & B_{2} \\
-\bar{A}_{2} & \bar{A}_{1} & -\bar{B}_{2} & \bar{B}_{1} \\
-\bar{B}_{1} & -\bar{B}_{2} & \bar{A}_{1} & \bar{A}_{2} \\
B_{2} & -B_{1} & -A_{2} & A_{1}
\end{array}\right) \mid \begin{array}{l}
{ }^{t} A \bar{A}-{ }^{t} \bar{B} B=I \\
{ }^{t} A \bar{B}+{ }^{t} \bar{B} A=0
\end{array}\right\},
$$

which is isomorphic to $S U^{*}(2 n)$ by mapping the element of the above given form to

$$
\left(\begin{array}{cc}
A_{1}+B_{2} & A_{2}-B_{1} \\
-\bar{A}_{2}+\bar{B}_{1} & \bar{A}_{1}+\bar{B}_{2}
\end{array}\right)
$$

With $\epsilon_{j}=1$ for $j=1, \ldots, p$, and $\epsilon_{j}=-1$ for $j=p+1, \ldots, n$, we then have $\sigma=\operatorname{Ad}\left(\operatorname{diag}\left(I_{p, q}, I_{p, q},-I_{p, q},-I_{p, q}\right)\right)$, with $I_{p, q}:=\operatorname{diag}(1, \ldots, 1,-1, \ldots,-1)$, and for the set of fixed points

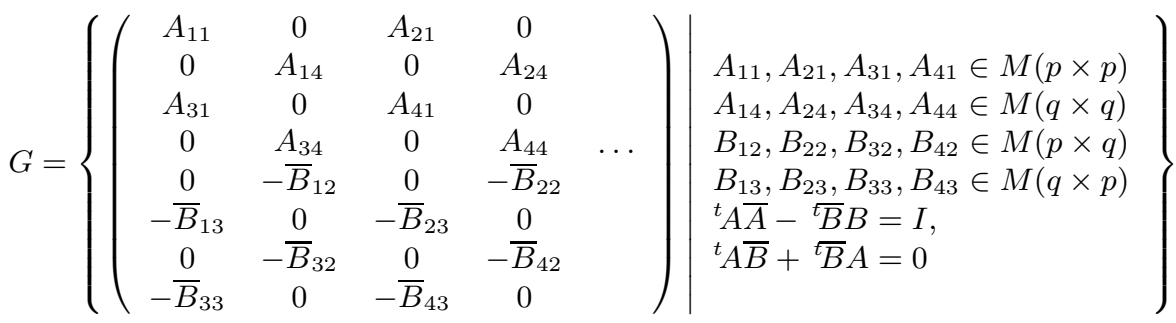

$$
\begin{aligned}
& \simeq\left\{\left(\begin{array}{rrrr}
A_{11} & A_{21} & B_{12} & B_{22} \\
A_{31} & A_{41} & B_{32} & B_{42} \\
-\bar{B}_{13} & -\bar{B}_{23} & \bar{A}_{14} & \bar{A}_{24} \\
-\bar{B}_{33} & -\bar{B}_{43} & \bar{A}_{34} & \bar{A}_{44}
\end{array}\right) \mid \cdots\right\}=U(2 p, 2 q)
\end{aligned}
$$

The image $\tilde{H}$ of $H$ is isomorphic to $S p(p, q)$ via conjugation with

$$
\left(\begin{array}{cccc}
i 1_{p} & 0 & 0 & 0 \\
0 & 0 & 1_{p} & 0 \\
0 & 1_{q} & 0 & 0 \\
0 & 0 & 0 & i 1_{q}
\end{array}\right)
$$

and for the causal compactification we find that

$$
\begin{aligned}
& S U(2 p, 2 q) / \tilde{H} \rightarrow \check{S}_{1}, \\
& \left(\begin{array}{cccc}
A_{11} & A_{21} & B_{12} & B_{22} \\
A_{31} & A_{41} & B_{32} & B_{42} \\
-\bar{B}_{13} & -\bar{B}_{23} & \bar{A}_{14} & \bar{A}_{24} \\
-\bar{B}_{33} & -\bar{B}_{43} & \bar{A}_{34} & \bar{A}_{44}
\end{array}\right) \tilde{H} \\
& \mapsto\left(\begin{array}{cccc}
A_{21} & B_{12} & -A_{11} & B_{22} \\
B_{13} & A_{24} & B_{23} & -A_{14} \\
A_{41} & B_{32} & -A_{31} & B_{42} \\
B_{33} & A_{44} & B_{43} & -A_{34}
\end{array}\right)\left(\begin{array}{cccc}
\bar{A}_{11} & -\bar{B}_{22} & \bar{A}_{21} & \bar{B}_{12} \\
-\bar{B}_{23} & \bar{A}_{14} & \bar{B}_{13} & \bar{A}_{24} \\
\bar{A}_{31} & -\bar{B}_{42} & \bar{A}_{41} & \bar{B}_{32} \\
-\bar{B}_{43} & \bar{A}_{34} & \bar{B}_{33} & \bar{A}_{44}
\end{array}\right)^{-1},
\end{aligned}
$$

with Bergman-ŠSilov boundary $\check{S}_{1}=\left\{Z \in M(2 n \times 2 n, \mathbb{C}) \mid{ }^{t} Z=-Z,{ }^{t} Z \bar{Z}=1\right\}$. 
7.3. The compactification of $S O_{0}(2, n-1) \times S O(2) /\left(O(1) \times S O_{0}(1, n-1)\right)$. We start with $G_{1}=S O(2, n+1)$ as in Example [5.2, again assume $n \geq 2$, and choose the same $E_{ \pm j}$ and $\eta$. We have to replace $H_{1}$ by $Z_{G_{1}}\left(X^{0}\right)$, since $G_{1 \mathbb{C}}$ is not simply connected. Applying Corollary 5.3 with $\epsilon_{1}=1=-\epsilon_{2}$, we have $\sigma=\operatorname{Ad}\left(I_{n, 2}\right)$ and correspondingly

$$
G=\left\{\left(\begin{array}{ccc}
A & B^{\prime} & 0 \\
C^{\prime} & D_{1}^{\prime} & 0 \\
0 & 0 & D_{4}
\end{array}\right) \in S L(n+3, \mathbb{R}) \mid \cdots\right\} \simeq S O_{0}(2, n-1) \times S O(2) .
$$

The centralizer $Z_{G}\left(X^{0}\right)$ is easily determined from $Z_{G_{1}}\left(X^{0}\right)$, as given in Example 5.2 to be

$$
Z_{G}\left(X^{0}\right)=\left\{\left(\begin{array}{ccccc}
\epsilon & 0 & 0 & 0 & 0 \\
0 & \epsilon a_{4} & B_{3}^{\prime} & 0 & 0 \\
0 & C_{2}^{\prime} & D_{1}^{\prime} & 0 & 0 \\
0 & 0 & 0 & \epsilon & 0 \\
0 & 0 & 0 & 0 & \epsilon
\end{array}\right) \in S L(n+3, \mathbb{R}) \mid \begin{array}{c}
\epsilon \in\{ \pm 1\}, \\
\left(\begin{array}{cc}
a_{4} & B_{3}^{\prime} \\
C_{2}^{\prime} & D_{1}^{\prime}
\end{array}\right) \in O(1, n)
\end{array}\right\},
$$

and therefore $G / Z_{G}\left(X^{0}\right) \simeq S O_{0}(2, n+1) \times S O(2) /\left(O(1) \times S O_{0}(1, n-1)\right)$.

7.4. Causal compactification of $S p(n, \mathbb{R}) \times S p(n, \mathbb{R}) / S p(n, \mathbb{R})$. We begin with $G_{1} \simeq S p(2 n, \mathbb{R})$ and the Cartan involution $\theta(g)={ }^{t} \bar{g}^{-1}$ as in Example 5.7 But as root vectors we choose now

$$
E_{j}=\frac{1}{2}\left(\begin{array}{cc}
0 & E_{j, j}+E_{j+n, j}+E_{j, j+n}+E_{j+n, j+n} \\
0 & 0
\end{array}\right)
$$

and

$$
E_{j+n}=\frac{1}{2}\left(\begin{array}{cc}
0 & -E_{j, j}+E_{j+n, j}+E_{j, j+n}-E_{j+n, j+n} \\
0 & 0
\end{array}\right),
$$

for $j=1, \ldots, n$, and $E_{-j}:=-\theta\left(E_{j}\right)$ for all $j$. With

$$
\sigma=\operatorname{Ad}\left(\begin{array}{cc}
I_{n, n} & 0 \\
0 & I_{n, n}
\end{array}\right)
$$

this involution commutes with $\theta$ and

$$
\eta=\operatorname{Ad}\left(\exp \left(\frac{\pi}{2} i X^{0}\right)\right)=\operatorname{Ad}\left(\begin{array}{cc}
0 & L_{n} \\
L_{n} & 0
\end{array}\right), \text { where } L_{n}:=\left(\begin{array}{cc}
0 & I_{n} \\
I_{n} & 0
\end{array}\right),
$$

giving the fixed point group

$$
\left.G=\left\{\begin{array}{rrrr}
A_{1} & 0 & B_{1} & 0 \\
0 & A_{4} & 0 & B_{4} \\
\bar{B}_{1} & 0 & \bar{A}_{1} & 0 \\
0 & \bar{B}_{4} & 0 & \bar{A}_{4}
\end{array}\right) \mid \begin{array}{l}
{ }^{t} A_{i} \bar{A}_{i}-{ }^{t} \bar{B}_{i} B_{i}=I \\
{ }^{t} A_{i} \bar{B}_{i}={ }^{t} \bar{B}_{i} A_{i} \\
i \in\{1,4\}
\end{array}\right\} \simeq S p(n, \mathbb{R}) \times S p(n, \mathbb{R}) .
$$

For the associated dual group we have

$$
G_{a}=\left\{\left(\begin{array}{rrrr}
A_{1} & 0 & 0 & B_{2} \\
0 & A_{4} & B_{3} & 0 \\
0 & \bar{B}_{2} & \bar{A}_{1} & 0 \\
\bar{B}_{3} & 0 & 0 & \bar{A}_{4}
\end{array}\right) \mid \cdots\right\} \simeq U(n, n)
$$


and, with $n$ the real rank of $U(n, n)$, condition (ii) of Theorem 5.1 can be verified immediately. Finally,

$$
H=\left\{\left(\begin{array}{rrrr}
A_{1} & 0 & B_{1} & 0 \\
0 & \bar{A}_{1} & 0 & \bar{B}_{1} \\
\bar{B}_{1} & 0 & \bar{A}_{1} & 0 \\
0 & B_{1} & 0 & A_{1}
\end{array}\right) \mid \begin{array}{l}
{ }^{t} A_{1} \bar{A}_{1}-{ }^{t} \bar{B}_{1} B_{1}=I, \\
{ }^{t} A_{1} \bar{B}_{1}={ }^{{ }_{B}} \bar{B}_{1} A_{1}
\end{array}\right\} \simeq S p(n, \mathbb{R}) .
$$

Putting things together, we get the causal compactification in the HarishChandra realization

$$
\begin{aligned}
& S p(n, \mathbb{R}) \times S p(n, \mathbb{R}) / \Delta(S p \times S p) \rightarrow \check{S}_{1}, \\
&\left(\left(\begin{array}{ll}
A_{1} & B_{1} \\
\bar{B}_{1} & \bar{A}_{1}
\end{array}\right),\left(\begin{array}{cc}
\bar{A}_{4} & \bar{B}_{4} \\
B_{4} & A_{4}
\end{array}\right)\right) \Delta(S p \times S p) \mapsto \quad\left(\begin{array}{cc}
B_{1} & -A_{1} \\
-A_{4} & B_{4}
\end{array}\right)\left(\begin{array}{cc}
\bar{A}_{1} & -\bar{B}_{1} \\
-\bar{B}_{4} & \bar{A}_{4}
\end{array}\right)^{-1},
\end{aligned}
$$

where the Bergman-ŠSilov boundary is $\check{S}_{1}=\left\{Z \in M(2 n \times 2 n, \mathbb{C}) \mid \bar{Z} Z=I,{ }^{t} Z=Z\right\}$, and $\Delta(S p \times S p):=\{(g, g) \mid g \in S p(n, \mathbb{R})\}$, the diagonal in $S p(n, \mathbb{R}) \times S p(n, \mathbb{R})$.

We note that our $\left(G_{1}, H_{1}\right)$ is a Cayley subspace of $\left(S U(2 n, 2 n), S L(2 n, \mathbb{C}) \cdot \mathbb{R}^{+} I\right)$. If we construct the causal compactification of $S(U(n, n) \times U(n, n)) / S U(n, n)$ as in section 7.1 starting from $G_{1}=S U(2 n, 2 n)$, then our $G / H$ is a causal subspace of $S(U(n, n) \times U(n, n)) / S U(n, n)$, and the causal compactification of $G / H$ constructed above is that of $S(U(n, n) \times U(n, n)) / S U(n, n)$ restricted to $G / H$.

7.5. The compactification of $S O^{*}(2 n) \times S O^{*}(2 n) / S O^{*}(2 n)$. For

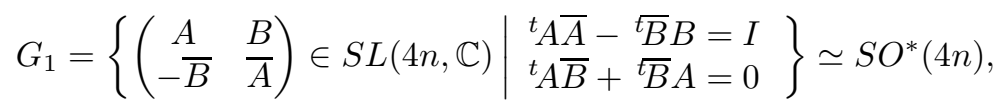

we choose $E_{j}=E_{j, j+3 n}-E_{j+n, j+2 n}$ and $E_{-j}=E_{j+3 n, j}-E_{j+2 n, j+n}$, for $j=$ $1, \ldots, n$. With $\theta$ and $\sigma$ as in section 7.4 we get

$$
\begin{aligned}
& \left.G=\left\{\begin{array}{rrrr}
A_{1} & 0 & B_{1} & 0 \\
0 & A_{4} & 0 & B_{4} \\
-\bar{B}_{1} & 0 & \bar{A}_{1} & 0 \\
0 & -\bar{B}_{4} & 0 & \bar{A}_{4}
\end{array}\right) \mid \begin{array}{l}
{ }^{t} A_{i} \bar{A}_{i}-{ }^{t} \bar{B}_{i} B_{i}=I \\
{ }^{t} A_{i} \bar{B}_{i}+{ }^{t} \bar{B}_{i} A_{i}=0 \\
i \in\{1,4\}
\end{array}\right\} \\
& \simeq S O^{*}(2 n) \times S O^{*}(2 n),
\end{aligned}
$$

and our root vectors give $X_{j}$ and $Y_{j}$, which satisfy condition (i) of Theorem 5.1 The involution

$$
\eta=\operatorname{Ad}\left(\exp i \frac{\pi}{2} X^{0}\right)=\operatorname{Ad}\left(\begin{array}{cc}
0 & J_{n} \\
-J_{n} & 0
\end{array}\right)
$$

makes $\left(G_{1}, H_{1}, \eta\right)$ into a Cayley space, and it commutes with $\sigma$. Also,

$$
H=\left\{\left(\begin{array}{rrrr}
A_{1} & 0 & B_{1} & 0 \\
0 & \bar{A}_{1} & 0 & \bar{B}_{1} \\
-\bar{B}_{1} & 0 & \bar{A}_{1} & 0 \\
0 & -B_{1} & 0 & A_{1}
\end{array}\right) \mid \begin{array}{l}
{ }^{t} A_{1} \bar{A}_{1}-{ }^{t} \bar{B}_{1} B_{1}=I, \\
{ }^{t} A_{1} \bar{B}_{1}+{ }^{t} \bar{B}_{1} A_{1}=0
\end{array}\right\} \simeq S O^{*}(2 n),
$$

so that for the causal compactification we now get, similarly to the last section,

$$
\begin{aligned}
& S O^{*}(2 n) \times S O^{*}(2 n) / \Delta\left(S O^{*} \times S O^{*}\right) \rightarrow \check{S}_{1}, \\
& \left(\left(\begin{array}{cc}
A_{1} & B_{1} \\
-\bar{B}_{1} & \bar{A}_{1}
\end{array}\right),\left(\begin{array}{cc}
\bar{A}_{4} & \bar{B}_{4} \\
-B_{4} & A_{4}
\end{array}\right)\right) \Delta\left(S O^{*} \times S O^{*}\right) \mapsto\left(\begin{array}{cc}
B_{1} & -A_{1} \\
A_{4} & B_{4}
\end{array}\right)\left(\begin{array}{cc}
\bar{A}_{1} & \bar{B}_{1} \\
-\bar{B}_{4} & \bar{A}_{4}
\end{array}\right)^{-1},
\end{aligned}
$$

with $\check{S}_{1}$ as in section 7.2 and $\Delta\left(S O^{*} \times S O^{*}\right)$ again the diagonal subgroup. 
7.6. The compactification of $S p(2 n, \mathbb{R}) / S p(n, \mathbb{C})$. The group $G_{1}=S U(2 n, 2 n)$ together with

$$
H_{1}=\left\{\left(\begin{array}{rrrr}
A_{11} & A_{21} & B_{12} & B_{22} \\
-\bar{A}_{21} & \bar{A}_{11} & -\bar{B}_{12} & \bar{B}_{12} \\
-\bar{B}_{13} & -\bar{B}_{23} & \bar{A}_{14} & \bar{A}_{24} \\
B_{23} & -B_{13} & -A_{24} & A_{14}
\end{array}\right) \in S L(4 n, \mathbb{C}) \mid \ldots\right\} \simeq S p(n, n),
$$

and the involution

$$
\tau(g)=\operatorname{Ad}\left(\begin{array}{cc}
J_{p} & 0 \\
0 & J_{p}
\end{array}\right)(\bar{g}),
$$

make up a compactly causal space $\left(G_{1}, H_{1}, \tau\right)$. Indeed, $G_{1} / H_{1}$ is isomorphic to the semisimple symmetric subspace contained in the weak compactly causal space $G / H$ constructed in section $\left[7.2\right.$. The real rank is $2 n$, and $E_{j}:=i E_{j, j+3 n}, E_{-j}:=$ $-i E_{j+3 n, j}, E_{j+n}:=i E_{j+2 n, j+n}$ together with $E_{-(j+n)}:=-i E_{j+n, j+2 n}$, for $j=$ $1, \ldots, n$, form a system of the second kind. Defining $\sigma$ as in Theorem 5.9 we find immediately that

$$
G=\left\{\left(\frac{A}{B} \quad \frac{B}{A}\right) \in S U(2 n, 2 n)\right\} \simeq S p(2 n, \mathbb{R})
$$

and

$$
H=\left\{\left(\begin{array}{rrrr}
A_{11} & A_{21} & B_{12} & B_{22} \\
-\bar{A}_{21} & \bar{A}_{11} & -\bar{B}_{22} & \bar{B}_{12} \\
\bar{B}_{12} & \bar{B}_{22} & \bar{A}_{11} & \bar{A}_{21} \\
-B_{22} & B_{12} & -A_{21} & A_{11}
\end{array}\right) \in S L(4 n, \mathbb{C}) \mid \cdots\right\} \simeq S p(n, \mathbb{C}),
$$

the last isomorphism being given by mapping an element of the given form onto

$$
\left(\begin{array}{cc}
A_{11}+i B_{22} & A_{21}-i B_{12} \\
-\bar{A}_{21}+i \bar{B}_{12} & \bar{A}_{11}+i \bar{B}_{22}
\end{array}\right) .
$$

Since the associated group $G_{a}:=G^{\sigma \theta} \simeq S O^{*}(4 n)$ has real rank $n$, the cited theorem gives us a causal compactification of $(G, H, \tau)$.

7.7. The compactification of $S O_{0}(2, q) \times S O(p+1) /\left(S O_{0}(1, q) \times O(p)\right)$. With $G_{1}=S O(2, n+1)$ we choose $\tau:=\operatorname{Ad}(\operatorname{diag}(1,-1, \ldots,-1,1, \ldots, 1))$, where -1 ranges from the second to the $(p+2)$-th position. Then

$$
H_{1}=\left\{\left(\begin{array}{cccc}
a_{1} & 0 & 0 & B_{2} \\
0 & a_{4} & B_{3} & 0 \\
0 & C_{2} & D_{1} & 0 \\
C_{3} & 0 & 0 & D_{4}
\end{array}\right) \in S L(n+3, \mathbb{R}) \mid \cdots\right\} \simeq S(O(1, p) \times O(1, q+1))
$$

and therefore $G_{1} / H_{1}$ is compactly causal ( $\mathrm{HO}$ 96, p. 89]). With the symmetric matrices

$$
\tilde{E}_{1}:=\frac{1}{2}\left(\begin{array}{ccccc}
0 & \ldots & -i & -1 & \\
\vdots & & -1 & i & \\
-i & -1 & & & \\
-1 & i & & & \vdots \\
& & & \ldots & 0
\end{array}\right) \text { and } \tilde{E}_{2}:=\frac{1}{2}\left(\begin{array}{ccccc}
0 & \ldots & i & -1 & \\
\vdots & & 1 & i & \\
i & 1 & & & \\
-1 & i & & & \vdots \\
& & & \ldots & 0
\end{array}\right) \text {, }
$$

where the first nonzero entries $-i$ and $i$ occur in the first row in the $(p+1)$-th column, and $\tilde{E}_{-j}:=-\theta\left(\tilde{E}_{j}\right)$, we have $\tau\left(\tilde{E}_{ \pm j}\right)=\tilde{E}_{\mp j}$. Similarly to Example 5.10 
we define $E_{ \pm j}:= \pm i \tilde{E}_{ \pm j}$ so that we get a system of the first kind. Applying Theorem 5.9 gives $\sigma=\operatorname{Ad}(\operatorname{diag}(1,1,-1, \ldots,-1,1, \ldots, 1))$, with -1 now from the third up to the $(p+3)$-th position, and

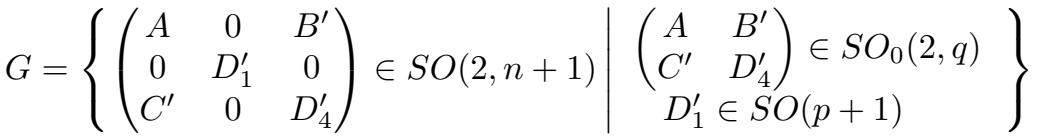

$$
\begin{aligned}
& \simeq S O_{0}(2, q) \times S O(p+1) .
\end{aligned}
$$

Intersecting with $H_{1}$ gives us

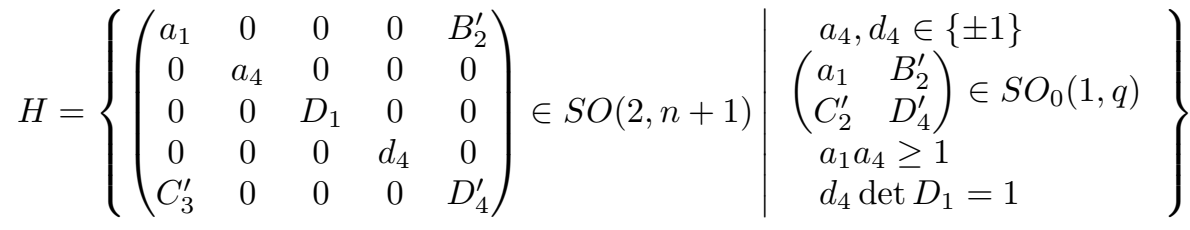

$$
\begin{aligned}
& \simeq S O_{0}(1, q) \times O(1) \times O(p),
\end{aligned}
$$

and the centralizer $Z_{G}\left(X^{0}\right)$ is formed by the elements with $a_{1} d_{4}=1$, i.e. $Z_{G}\left(X^{0}\right) \simeq$ $S O_{0}(1, q) \times O(p)$. Therefore we have a causal compactification for $G / Z_{G}\left(X^{0}\right) \simeq$ $S O_{0}(2, q) \times S O(p+1) /\left(S O_{0}(1, q) \times O(p)\right)$.

\section{REFERENCES}

[Be] W. Bertram, Algebraic structures of Makarevič spaces I, Transformation Groups 3 (1988), 3-32. MR 99c:32047

[FÓ] F. Betten and G. Ólafsson, Causal compactification and Hardy spaces for spaces of Hermitian type, Pacific J. Math. 200 (2001), 273-312. CMP 2002:05

[He 78] S. Helgason, Differential Geometry, Lie Groups and Symmetric Spaces, Academic Press, New York 1978 MR 80k:53081

[HÓ 96] J. Hilgert and G. Ólafsson, Causal symmetric spaces, The Geometry and Harmonic Analysis, Perspectives in Math. 18, Academic Press, New York 1996 MR 97m:43006

[HÓØ] J. Hilgert, G. Ólafsson, and B. Ørsted, Hardy spaces on affine symmetric spaces, J. reine angew. Math. 415 (1991), 189-218 MR 92h:22030

[Ka 87] S. Kaneyuki, On orbit structure of compactification of para-hermitian symmetric spaces, Japan J. Math. 13 (1987), 333-370 MR 88m:53094

[Ka 89] S. Kaneyuki, On the causal structures of the Šlov boundary of symmetric bounded domains, in "Prospects in Complex Geometry", Springer LN 1468, New York 1989, pp. 127-159 MR 92h:32049

[Ko 93] K. Koufany, Semi-groupe de Lie associé à une algèbre de Jordan euclidienne, thesis, Université de Nancy I, 1993

[KØ96] K. Koufany and B. Ørsted, Function spaces on the Ol'shanskiて semigroup and the Gelf'and-Gindikin program, Ann. Inst. Fourier 46 (1996), 689-722 MR 97k:22021

[KØ97] K. Koufany and B. Ørsted, Hardy spaces on two sheeted covering semigroups, J. Lie Theory 7 (1997), 245-267 MR 98k:22060

[KW 65] A. Korányi and J. A. Wolf, Realization of Hermitian symmetric spaces as generalized half planes, Ann. of Math. 81 (1965), 265-288 MR 30:4980

[Ma 73] B. O. Makarevič, Open symmetric orbits of reductive groups in symmetric $R$-spaces, Math. USSR Sbornik 20 (1973), 406-418 MR 50:1170

[Ma 79] T. Matsuki, The orbits of affine symmetric spaces under the action of minimal parabolic subgroups, J. Math. Soc. Japan 31 (1979), 331-357 MR 81a:53049

[Ma 82] T. Matsuki, Orbits on affine symmetric spaces under the action of parabolic subgroups, Hiroshima Math. J. 12 (1982), 307-320 MR 83k:53072

[Mo 97] V. F. Molchanov, Holomorphic discrete series for hyperboloids of Hermitian type, J. Funct. Anal. 147 (1997), 26-50 MR 99b:22026

[Ól 90] G. Ólafsson, Causal symmetric spaces, Mathematica Gottingensis 15, 1990 
[ÓØ 97] G. Ólafsson and B. Ørsted, Causal compactification and Hardy spaces, Trans. AMS 351 (1999), 3771-3792 MR 99m:22005

[Va 84] V. S. Varadarajan, Lie Groups, Lie Algebras and Their Representations, 2nd edition, Springer, New York 1984 MR 85e:22001

[Wa 72] G. Warner, Harmonic Analysis on Semi-Simple Lie Groups I, Springer Grundlehren der mathematischen Wissenschaften 188, Berlin 1972 MR 58:16979]

[Wo 72] J. A. Wolf, Fine Structure of Hermitian Symmetric Spaces, in "Symmetric Spaces", pp. 271-357, Dekker, New York 1972 MR 53:8516

Mathematisches Institut, Universität Göttingen, Bunsenstrasse 3-5, D-37073 Göttingen, Germany 